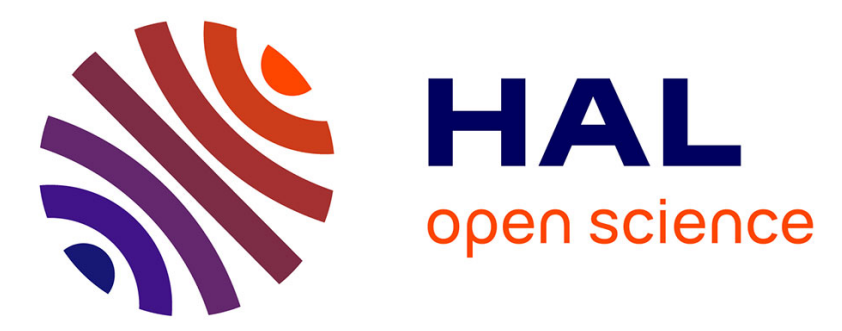

\title{
A Survey on Health Monitoring Systems for Health Smart Homes
}

\author{
Haider Mshali, Tayeb Lemlouma, Maria Moloney, Damien Magoni
}

\section{To cite this version:}

Haider Mshali, Tayeb Lemlouma, Maria Moloney, Damien Magoni. A Survey on Health Monitoring Systems for Health Smart Homes. International Journal of Industrial Ergonomics, 2018, 66, pp.26-56. 10.1016/j.ergon.2018.02.002 . hal-01715576

\section{HAL Id: hal-01715576 https://hal.science/hal-01715576}

Submitted on 22 Feb 2018

HAL is a multi-disciplinary open access archive for the deposit and dissemination of scientific research documents, whether they are published or not. The documents may come from teaching and research institutions in France or abroad, or from public or private research centers.
L'archive ouverte pluridisciplinaire HAL, est destinée au dépôt et à la diffusion de documents scientifiques de niveau recherche, publiés ou non, émanant des établissements d'enseignement et de recherche français ou étrangers, des laboratoires publics ou privés. 


\title{
A Survey on Health Monitoring Systems for Health Smart Homes
}

\author{
Haider Mshali ${ }^{\mathrm{a}}$, Tayeb Lemlouma ${ }^{\mathrm{b}, *}$, Maria Moloney $^{\mathrm{c}}$, Damien Magoni $^{\mathrm{a}}$ \\ ${ }^{a}$ Université de Bordeaux - LaBRI, Talence, France \\ ${ }^{b}$ Université de Rennes 1 - IRISA, Lannion, France \\ ${ }^{c}$ University College Dublin - PEL, Dublin, Ireland
}

\begin{abstract}
Aging population ratios are rising significantly. Health monitoring systems (HMS) in smart environments have evolved rapidly to become a viable alternative to traditional healthcare solutions. The aim of HMS is to not only reduce costs but to also provide timely e-health services to individuals wishing to maintain their independence. In this way, elderly people can avoid, for as long as possible, any interaction with healthcare institutions (e.g. nursing homes and hospitals), which in turn reduces pressure on the health system. To fully realise this vision of seamless e-health services supporting people in need of them, a number of challenges that need further investigation still exist. To this end, we provide an overview of the current state of the art for smart health monitoring systems. We review HMS in smart environments from a general perspective and with a particular focus on systems for the elderly and dependent people. We look at the challenges for these systems from the perspective of developing the technology itself, system requirements, system design and modelling. We present a consolidated picture of the most important functions and services offered by HMS for monitoring and detecting human behaviour including its concepts, approaches, and processing techniques. Moreover, we provide an extensive, in-depth analysis and evaluation of the existing research findings in the area of e-health systems. Finally, we present challenges and open issues facing the smart HMS field and we make recommendations on how to improve future systems.
\end{abstract}

Keywords: Health Monitoring System, Health Smart Home, Health-care, e-Health, Context-aware, Sensor networks

\section{Introduction}

Population aging is happening faster than ever before. According the United Nations Population Fund (UNFPA) [1], the global number of people aged 60 or older will rise to 2 billion by 2050. Added to this, a report issued by the World Health Organization (WHO) stated that there was a shortage of about 7.2 million healthcare workers in 2013, and this is estimated to reach 12.9 million by 2035 [2]. Currently, a significant proportion of the elderly population suffers from an age-related health issue such as Alzheimer's disease, dementia, diabetes, cardiovascular disease, osteoarthritis or other chronic diseases. These common diseases, coupled with the naturally occurring progressive decline in physical and cognitive skills of elderly people prevent many from living independently, in their own homes. Recent advances in communications and computing technologies, along with advances in ambient intelligent technologies, such as sensors, have resulted in a rapid emergence of smart environments. One such example is the so-called Health Smart Home (HSH). HSHs are smart environments where a context-aware health monitoring system is set up. They have attracted considerable interest in recent years. HSH emerged as a promising solution to the challenge of an increasing aging population.

\footnotetext{
* Corresponding author.
}

They have the potential to provide e-health services to meet the needs of this growing population. In particular, HSH systems monitor and evaluate any health condition that the elderly subject may have, as well as monitoring how they carry out their daily activities. These systems not only allow the elderly to live independently for longer but they also have the potential to make healthcare services more sustainable by reducing the pressure placed on the overall health system by the elderly and dependent individuals.

To achieve a truly health smart home, a number of challenges still exist in many aspects of the development procedure. Such challenges include the remote monitoring of the environment (data acquisition about the subject, their environment, etc.), the communication technology needed for the environment (reliability of data transmission in real-time), the existence of intelligent processing systems (analysis, making relevant decisions, etc.) and the provision of context-aware services. Consequently, to meet these challenges, further research is required to improve the design of efficient health monitoring systems for HSH.

In order for health monitoring systems (HMS) to proactively help individuals in terms of required assistance and services, it needs full access to a set of contextual information. The success of these systems lie in the ability to 
collect and process data to understanding a subject's surroundings, so that contextual services can be delivered to them. What we mean by contextual services in the field of healthcare is a set of continuous processes that automatically acquire a subject's information (e.g. behavioural, physiological, and environmental), and as a result of this acquisition, are able to provide and adapt the services accordingly. The context and surroundings of the elderly cannot be easily confined to a simple level of data acquisition from sensors but, instead, should be enriched by data that has been integrated and inferred from the environment, using several approaches and techniques, to enrich existing knowledge about the subject. Context-awareness facilitates a better understanding of the health conditions of the subject being monitored, by identifying behaviour patterns and making more precise inferences about the subject's situation and their environment. Numerous survey studies such as [3 [8] have been carried out in the literature. These studies have mainly focused on smart home perspectives [3], health smart homes with limited information about design issues and challenges [4], wearable sensor-based systems for health monitoring [5], and health informatics and healthcare applications for the elderly from various perspectives [6] [7] [].

In this review paper, we present a comprehensive study of context-aware computing in healthcare for the elderly. Our objective is to highlight the current technologies, as well as the requirements and the challenges in the design of HMS in smart environments. We present a set of guidelines to aid interested parties in understanding the issues that need to be addressed to improve e-healthcare services. We discuss in detail the components of HMS, and provide a better understanding of the context of monitored subjects, and classify the main activities that should be considered to process HSH scenarios. We attempt to present a consolidated picture of the most important functions and services offered by HMS for monitoring and detecting human behaviour including its concepts, approaches, and processing techniques. We provide a survey of extant research and we evaluate the state of the art for healthcare applications and monitoring systems. We also discuss the benefits and some research challenges for future healthcare applications.

The rest of the paper is organized as follows: Section 2 gives the definition and meaning of context data and context-aware computing and discusses how these concepts are used in healthcare monitoring. Section 3 presents an overview of health smart homes and describes the design issues regarding healthcare monitoring systems. Section 4 presents the general architecture for healthcare monitoring systems. Section 5 presents modelling and reasoning methods and learning algorithms used to analyse and understand human behaviour. This is coupled with a classification of human activities. These activities are considered in HMS scenarios and used in different functionalities offered by HMS. Section 6 summarizes several applications under three categories related to the elderly and patients in a smart environment. Section 7 identifies a number of research challenges and addresses open research issues in this area. Finally, Section 8 concludes the review.

\section{Context-Aware Applications in Healthcare}

Many definitions of the context concept have been proposed in the literature. According to [9] context represents any information that can be used to characterize the situation of an entity. An entity includes a person, a place or an object. The authors of [10] provide a distinction between two types of information: raw data and context information. Raw data, also called low-level context data, is unprocessed data taken directly from the source such as a sensor. In e-health, such data may represent a person's vital signs, environmental parameters (e.g. temperature, humidity, and sound), or personal movements, etc. Note that the acquired raw data is worthless if it is not well interpreted and understood by the HMS. The second category is context information generated by processing raw data, i.e. by applying consistency validation or metadata enrichment. It refers to extracting high-level information such as behaviour patterns, or a subject's activity, etc.

Context plays a significant role in health monitoring, mainly for two reasons. Firstly, for smart environments, evaluating the monitored subject regarding their health conditions and their performances in achieving their activities of daily living (ADL) relies on understanding the subject's surroundings. For instance, changes in the subject's vital signs data should be correlated with the subject's current situation in order to achieve a better understanding of the subject's condition. For example, the system's interpretation of the subject's cardio activity should be linked to the nature of the subject's current activity, which can differ substantially for the housekeeping activity compared to the watching $T V$ activity. A high bathroom activity may refer to the risk of diabetes, a high recording of sleep disorders could imply hepatitis C [11], etc. Secondly, context can be useful in making HMS more efficient and optimal. In HSH, data regarding the subject's context (behavioural, physiological, and environmental) is often collected using various sensors and devices. A continuous processing and fusion of such data in HMS requires a huge amount of computation. The core to ensuring HMS effectiveness is to make sure that the necessary sensors are appropriately selected and that the data is appropriately and accurately analysed (i.e. the collection and analysis of highly relevant contextual information) [12].

Context has been broken down into various dimensions depending on the different perspectives in the field. Each context dimension is used to define a piece of information. For example, the authors in [13] considered location, environment, identity and time when defining context. In [14], context data is collected based on answers to the following three questions: where, who and what? In [15], context refers to location, identity, activity and time. These dimensions act as the primary layer of context when characterizing a given situation. Then, other related 
information can be used to refine the description of the context. For example, given a person's identity, we can collect other related dimensions such as phone numbers, addresses, other nearby people in the environment, etc. The primary pieces of the context should answer the following five $W$ questions Who, What, Where, When, and Why. These questions were identified in [16] as a minimal set of information necessary to understand context and situations. For instance, identifying the person and their location along with identifying one activity performed at a specific time could provide useful information for health monitoring systems. The evaluation of the subject's conditions will, consequently, depend on the accuracy of the current context information. A lying position in the bedroom at night refers to a normal situation, while the same position in the middle of the day for an extended period could indicate an abnormal situation.

Several definitions of context-awareness can be found in the literature. We will focus on definitions that give a clear idea of how this concept can be used in health monitoring systems. Context-awareness allows for the automatic provision of information or for the system to take action according to the subject's current context and needs [17]. According to [18], context-awareness refers to applications capable of monitoring inputs from sensor devices and selecting the suitable context according to the subject's needs or interests. In [15], a system is qualified as context-aware if it uses context to provide relevant information and services to the subject. In contrast, from the pervasive healthcare systems perspective, context-aware computing systems consider the application's ability to adapt to changing circumstances and respond according to the context of use [19].

Generally, context-aware computing systems are usually designed and implemented for a specific purpose and, thus, are focused on finding solutions to specific problems. As a result, no standards or guidelines exist for building such systems. The core difference between each system lies in the levels of context-awareness each system possesses. In context-aware systems, this degree is usually called level which in turn has been defined in various ways [20]. Therefore, designing an optimal contextaware system for e-health is still an open issue with several challenges. In spite of this complexity, the analysis of hospital-based projects [21] reveals that several efforts have been made when considering context-aware computing in healthcare applications. There are, however, still many limitations and drawbacks with context awareness e-health services. For example, the lack of any recommendations regarding the contextual functional needs of the subject, the gap between fundamental context representations and actual context awareness prototypes and the difficulty of building an efficient system to simulate the human perspective.

Broadly speaking, context-awareness systems have many components that are responsible for several functions including the representation, management, reasoning, and analysis of context information. Different types of contextual information are obtained from various sources (e.g. sensors) deployed in the monitoring area network of the HSH. Data models are used to represent and store context data. A clear insight into the monitored subject's lifestyle and environment can be achieved by extracting high levels of contextual information and by aggregating and interpreting the information. Consequently, the system processes the analysed context for e-health contextaware applications and allows the system to dynamically determine the subject's real needs in terms of assistance and services.

\section{Overview of Health Smart Homes}

Health Smart Homes (HSH) and Healthcare Monitoring Systems (HMS) are an integration of ubiquitous computing and communication technologies. These systems have emerged as a promising solution to provide e-health services that match the context and real needs of subjects. In this realm, several studies and solutions have been proposed to address diverse aspects of these systems. In general, these solutions share common goals and properties. Their main objective is to provide a smart environment where the system monitors and evaluates the health conditions of subjects and provides them with timely e-health services.

These solutions include three main components: sensing, communication, and the processing system. Most existing pervasive health systems have the ability to interact with different categories of subjects (e.g. patients, elderly, and disabled) using one or more types of sensors. Sensors are responsible for data acquisition and are either stationary sensors deployed in domestic settings or wearable sensors carried by the subject. Both of these two categories of sensors collect the subject's contextual information in a constant or periodic way. Sensors relay data through, for instance, an access point or a base station to a home server or portable devices via available network technologies. Home servers and/or portable devices act as gateways. A gateway is a common element used in $\mathrm{HSH}$ and is usually seen as the network coordinator that provides remote access control and acts as the bridge between the local area network (LAN) and the wide area network (WAN) such as the Internet, cellular or fixed telephony networks.

At the application level, the actors of a health monitoring system (patients or health providers) use a graphical user interface (GUI). Depending on the complexity of the system, GUIs can be used in monitoring and evaluating the subject's health conditions and can notify caregivers in the case of an abnormality or an emergency situation. Diverse parameters and heterogeneous data collected from sensors can be involved, such as from the environment (e.g. temperature, humidity, and sound detection), movement and location tracking (e.g. gesture and pressure), and vital 


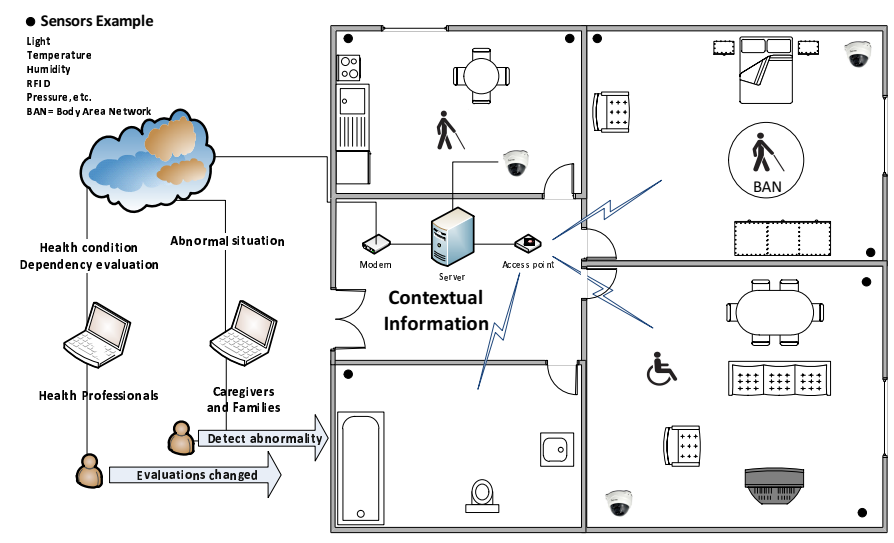

Figure 1: General overview of a Health Monitoring System (HMS). The monitoring system applies either a regular evaluation of the person's condition, or a rapid intervention in the case of detection of abnormalities.

signs (e.g. heart rate, oxygen saturation, and blood pressure). This data provides a low-level view of the context of the person's health status, their surroundings and living environment. According to the system architecture, hardware capabilities (sensors, connections, and gateways) and methods used in processing the data, the system can gain a high-level view of the context. In complex and intelligent applications, this high-level view is used for many reasons such as the classification of diseases, predicting health conditions, pattern detection in human behaviour, etc. Fig 1 shows a general overview of an HMS.

HSHs are based on a common set of components responsible for sensing, communicating, and processing data. However, existing systems can be differentiated based on the following three aspects: (i) the target, (ii) the capabilities, and (iii) employed methods. Firstly, depending on the target application and population, the differences between existing $\mathrm{HSH}$ projects lie in the consideration of the subject's needs and requirements, which can lead to a focus on a set of limited context dimensions. Many projects reduce context to a single parameter or objective. For instance, projects focus on fall detection and location tracking or they monitor and evaluate a single type of daily activity (e.g. sleeping), a certain cognitive disease (e.g. Alzheimer's) or a certain biological disease (e.g. heart disease).

Secondly, the capability of a HSH is related to its processing, storage, mode of communication, bandwidth, and energy management of the devices used in the sensor network. Based on these capabilities, which increase from lower to higher levels, the data can be processed at many levels. Overall, the most common structural components of a sensor network used in healthcare monitoring systems are presented in (Fig,2). The data collection follows a bottom-up approach. In such a structure, raw numeric data is generated by terminal sensor nodes deployed in a designated area. Each sensor node gathers and transmits data directly to a gateway or base station called the sink node (static or mobile sink), which in turn is connected with the gateway. Finally, if necessary, data reaches the WAN for external exchanges. Sensors and devices in lower levels perform a certain amount of basic processing on the captured data, which can reduce the amount of transferred data and prevent network congestion. However, due to the hardware and software limitations of devices in terms of processing, memory, and energy, the majority of data processing is done at higher levels. Therefore, the system capabilities represent a key aspect for determining the smartness of the health monitoring system.

Finally, there are different methods and algorithms that can be used in HSH, such as learning-based and reasoningbased approaches. The choice of methods depend on several considerations, such as the amount and nature of collected data (e.g. using a sensor-based or vision-based data), the monitoring methodology, and the application requirements. In the following subsections, we briefly present the most important components required in HMS, in terms of sensor systems, communication gateways, and applications.

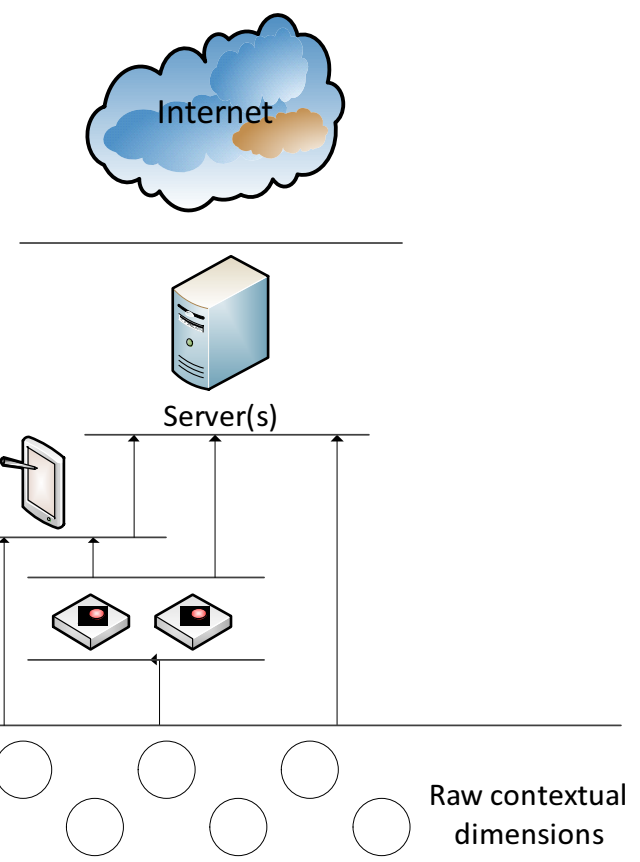

Figure 2: The structure of a sensor network in HMS.

\subsection{Sensor Systems}

Data acquisition happens first in the health monitoring environment. In this stage, various sources are used to gather the information related to the physical status of the subject, their behaviour, their environment, the activities they have performed, etc. Context-aware e-health systems need to have full visibility of the subject's context. This requires using all the available sources of data collection. Data sources may be classified according to their collection method (direct and indirect), event type (frequency mode), source type (physical or virtual) and sensor data. 
The collection of data can be categorised into two categories depending on how the system gathers data, either directly or indirectly. Direct methods acquire data from hardware and sensors attached locally without intermediaries. These sensors capture information constantly and, in most instances, relay data wirelessly using numerous communication protocols to a home server or a coordinator. Indirect methods refer to data acquisition using a middleware-based infrastructure where the system collects sensor data from additional software or hardware sources. In this category, data might come from storage servers, databases, or RSS (Really Simple Syndication) feeds, etc.

With regards to data collection, the frequency of receiving data plays an important role in overall system performance. Data can be generated based on three event types: constant, interval or instant. Constant events ensure that data is continuously transmitted. For example, using an IP camera which sends a continuous video stream. In instant events, acquired data is instantly sent when a certain event occurs. For example using a light switch or a door detection sensor. Interval events imply that the data is sent periodically following a uniform time interval. For example, sensing and sending heart performance every 20 seconds with an ECG sensor.

In HMS, physical sensors are the most common type of sensors used to generate and retrieve raw sensor data including data relating to the subject and their environment. Virtual sources refer to other data sources, such as existing health records, historical data (e.g. the person's behaviour and dependency level), the subject's social media network, the profiles of other individuals involved in the overall ehealth ecosystem such as patients, the elderly, disabled and caregivers including professionals, nurses, volunteers, etc.

Sensor data can have different formats, such as numerical, categorical, graphics, video, etc. Based on these formats and sensor types, health monitoring can be classified into two approaches: vision-based and sensor-based approaches. The vision-based approaches are based on visual sensors, such as video cameras for movement and gesture recognition, while sensor-based approaches use a wide range of emerging sensors and technologies for health and biomedical monitoring. Several devices, such as MicaZ, Telos Sky, and Tmote Sky equipped with a set of sensors (e.g. light, temperature, humidity, acceleration, magnetic, etc.) were used in different studies and projects [22] 23]. Table 1 summarizes some recent sensor nodes that can be used in this context [24].

Table 1: Physical sensor nodes 24].

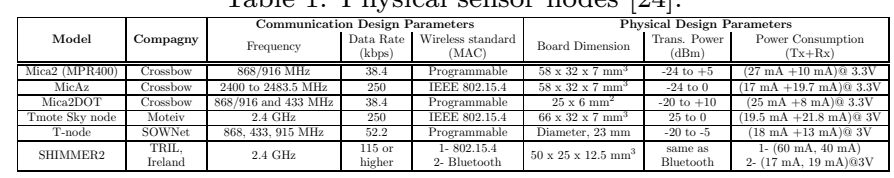

Nowadays, systems and projects for healthcare monitoring rely on a set of common components and use stan- dard or commercial sensors for the purpose of gathering raw data to monitor individuals and their environment. According to the research projects surveyed in this paper, there are three main classes of interconnected networks, which are often used in this realm: Personal Sensor Networks (PSN), Body Sensor Networks (BSN) and Multimedia Devices (MD). Selected sensors and devices are integrated into home objects and infrastructure and connected using network technologies. Each sensor is responsible for one or more task at the same time.

PSNs are used to detect human daily activities and measure conditions in the subject's environment. BSNs are used to monitor vital signs and health conditions by measuring physiological parameters and detecting ambulatory activities. Finally, more contextual information related to human activity is collected via MD to monitor movement, environmental changes and to increase the interaction between the monitored subject and the e-health application. An overview of sensor networks in HMS is depicted in Fig. 3. Table 2 describes and provides the purpose for the three network categories, including the sensors and devices that are used in HMS.

Table 2: Health Monitoring Devices and Sensors.

\begin{tabular}{|c|c|c|c|}
\hline Category & Name & Purpose & Data Format \\
\hline \multirow{10}{*}{$\mathrm{PSN}^{1}$} & PIR & Motion detection & Categorical \\
\hline & RFID & Persons and objects identification & Categorical \\
\hline & Pressure & $\begin{array}{l}\text { Identify location } \\
\text { Inseritin }\end{array}$ & Numerical \\
\hline & Ultrasonic & Tracking location and posture & Numerical \\
\hline & Contact switches & Open/close door detection & Categorical \\
\hline & Light & Use of light and its intensity & Time series \\
\hline & Temperature & Measure room temperature & Time series \\
\hline & Weight & Elderly weight & Numerical \\
\hline & Humidity & Measure room humidity & Time series \\
\hline & & On/off and measure power consumption & Numerical \\
\hline \multirow{11}{*}{$\mathrm{BSN}^{2}$} & Accelerometer & Measure acceleration, fall detection, location and posture & Time series \\
\hline & Gyroscopes & Measure orientation, motion detection & Time series \\
\hline & & Motion detection and location tracking & Categorical \\
\hline & ECG & Monitor cardiac activity & Analog signal \\
\hline & EEG & Measure of brain waves & Analog signal \\
\hline & EOG & Monitor eye movement & Analog signal \\
\hline & EMG & Monitor muscle activity & Analog signal \\
\hline & & Heart rate and blood velocity & Analog signal \\
\hline & Pulse oximeter & Measure blood oxygen saturation & Analog signal \\
\hline & Blood pressure & Measure blood pressure & Numerical \\
\hline & SKT & Skin temperature & Numerical \\
\hline \multirow{4}{*}{$\mathrm{MD}^{3}$} & & & \\
\hline & Microphone & Voice detection & Audio \\
\hline & Speakers & Alerts and instructions & Audio \\
\hline & & Visual information & Audio, video \\
\hline
\end{tabular}

${ }^{1}$ Personal Sensor Network, ${ }^{2}$ Body Sensor Network, ${ }^{3}$ Multimedia Devices

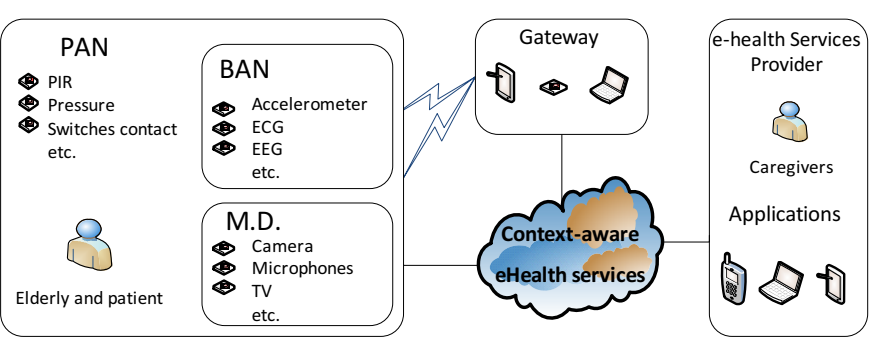

Figure 3: An overview of sensor networks used in HMS.

\subsubsection{Personal Sensor Networks (PSNs)}

PSNs or environmental sensors are responsible for capturing and retrieving contextual data regarding the subject and their environment. PSNs can be placed in a living 
environment or attached to different home objects to detect the subject's activities. Such objects could be a sofa, table, bed, chair, or floor containing pressure sensors. A subject's performance level regarding their daily living activities (ADL) can be measured by observing them in their environment and their personal interactions with household objects in a specific location. For instance, if a motion sensor identifies the current user location as kitchen and one sensor of cooking objects (e.g. gas, oven, toaster, or hob) is $O n$, and there is a water usage or the refrigerator's door is open (using a mixer tap sensor or contact switches), then sensed data indicates that the ADL activity of meal preparation is taking place. Environmental sensors can provide rich contextual information to detect the daily activities and observe human behaviour in $\mathrm{HSH}$. Passive infrared (PIR) sensors are most frequently used for detecting the person's presence [25] and ADL [26]. The use of radio-frequency identification (RFID) includes active and passive tags attached to objects and readers worn by the subject. RFID is used to identify users and objects of the HSH environment [27].Pressure [28] and ultrasonic [29] sensors can be attached to home objects to track their locations and therefore the movements of the user. Contact switches are used to detect the subject's interactions with other objects in the space (e.g. door, window, fridge, etc.). Environmental sensors are used for additional dimensions such as light, temperature, humidity. They are deployed in different places to monitor environmental conditions and identify daily activities. Power sensors are used to measure and manage energy consumption and to detect the usage of electrical devices using On/Off events. The usage of electrical appliances such as microwaves, water kettles, toasters, room heaters and televisions can also be used to detect activity and to further refine behaviour information [30].

\subsubsection{Body Sensor Networks (BSNs)}

BSNs use wearable sensors carried by monitored subjects such as the elderly and patients. These sensors are used in HMS to provide a continuous flow of information about real-time health conditions. They are often embedded into accessories such as clothes, belts, watches, or glasses. BSN often use inertial measurement units such as accelerometers for detecting ambulatory activities or vital sign devices such as heart rate sensors for monitoring the health condition of the subject. Accelerometers and gyroscopes are the most common inertial sensors used to monitor movements and body postures such as standing, sitting, and walking. Accelerometer sensors measure the acceleration values that are typically based on three-axis accelerometers positioned on well-defined locations on the human body. The authors of [31] used four accelerometers attached at the chest, left under-arm, waist and thigh to monitor and recognize five activities, standing, sitting, lying, walking and transition.

In [32], a human activity recognition (HAR) method is proposed using four wearable accelerometers mounted on the left/right forearms and left/right shins of subjects. The proposed system monitored the performance of ten daily activities and gym exercises, including standing straight, sitting on a chair, walking, jogging, and weight lifting. Authors in [33] monitored twenty-six events and activities using a single accelerometer and gyroscope sensor. Gyroscope sensors are typically used together with accelerometers for movement detection. In 34], accelerometer and gyroscope sensors were mounted on the right arm wrist and right foot of the subject in order to collect linear accelerations and angular rates of motion and thus to recognize six activities, walking, standing, writing, smoking, jacks, and jogging. Similarly, a fall detection system for the elderly was proposed in [35] using accelerometers and gyroscopes. Global positioning systems (GPS) can also be used as wearable sensors to monitor location-based activities in an open or mobile environment. For instance, to find out locations or predict movement across multiple subjects 36] or to learn and infer the subject's mode of transportation based on GPS data logs 37.

In HMS, several biosensors are used to monitor the vital signs of patients and the elderly, such as heart rate, oxygen saturation, blood pressure, blood glucose, body temperature, weight, etc. Wearable sensors have been embedded into watches [38] 39], shirts 40], belts [41], etc. Such sensors provide real-time physiological information related to the health condition of the monitored subject. Various biosensors are used in HMS, such as electrocardiography sensors (ECG) used to monitor cardiac activity, electroencephalography sensors (EEG) used to monitor brain activity, electromyography sensors (EMG) used to monitor muscle activity, and electrooculography sensors (EOG) used to monitor eye movements. Pulse oximeters are used to measure the oxygen level of the blood (i.e. oxygen saturation) while photoplethysmography sensors (EPG) are used to monitor the rate of blood flow. Other biomedical parameters can also be evaluated such as using $\mathrm{CO}_{2}$ gas sensors to evaluate gaseous carbon dioxide levels to monitor respiration.

To date, the majority of selected devices do not offer an interface for data relay. In fact, most existing studies and projects did not attempt to improve the linking of these devices to their infrastructure. The objective was usually to transform this category of sensors into context data sources connected to the network and to be able to directly and automatically collect data. Although there are several protocols and algorithms that have been proposed in wireless sensor networks, there are no well-suited features or requirements for body area networks (BANs) [5]. Parameters like the number of sensors, data rate, mobility, latency, communication, and transmission are selected based on the application type and the needs of the subject. In addition, energy consumption and battery life still pose major challenges for devices in such networks. 


\subsubsection{Multimedia Devices (MDs)}

Widespread use of home appliances, enriched by a set of sensors, helps to create an interactive healthcare environment. Integrated appliances can be electrical or electronic devices that fulfil several functions at home. Devices such as cameras, microphones, telephones, speakers and TV sets create a platform for the exchange of data between the subject and the system. These devices increase the subject's interactions with health applications and represent either new sources for gathering contextual information or a means for providing guidance and counseling [42].

Multimedia-based approaches encompass visual and audio sensing devices (e.g. cameras and microphones). Soundbased detection methods can be used to monitor some activities of daily living [43].Vision-based methods have a much wider scope and are used for posture recognition 44], human presence [45], movement and fall detection [46], and the monitoring of complex activities [47].

There has been disagreement with regards to the use of multimedia-based applications for such approaches. Although multimedia-based approaches provide rich contextual information, they suffer from computational costs and privacy issues. Data acquisition varies from one sensor to another. The set of heterogeneous sensors that are used in such approaches only provides basic raw data. Low-level data is imperfect, uncertain in nature and less meaningful. Therefore, further development is needed to build a high-level approach for providing healthcare services.

\subsection{Gateway and Communication Technologies}

A gateway is a key component for HSH and HMS systems. Its main functionalities include network interconnection, network management, and application management. Gateways are responsible for the coordination between heterogeneous sensors and their connection to the Wide Area Network (WAN).

The ability of a gateway to perform data processing plays a major role in preventing network congestion by reducing the amount of transferred data either directly or indirectly. The aim is also to benefit from the software and hardware capabilities of the gateway for advanced data analysis and processing. Intelligent algorithms can be used for data fusion and transmission control of data captured by heterogeneous sensors, for instance, by requesting a capture only when it is necessary for the purpose of a given service. Section 5 details numerous methods and techniques for using the results of intelligent algorithms and reasoning approaches. A gateway can host local services and acts as a reactive system which faces some simple events such as temperature variation and smoke detection. More complex reactive and proactive services can be provided by analyzing the subject's context and environment in HSH. These services provide direct guidance to the subject and are able to send alert messages to caregivers. In most cases, the gateway is implemented through a fixed device such as a local homebox. However, the gateway can be a mobile device like a smartphone, a tablet, or a local smart sensor node placed in the environment or mounted on a computer server. From a HMS overview perspective, the gateway relays data by using different network systems such as fixed telephony networks, WiFi, cellular networks, satellite networks, etc. The available networking technologies are combined to serve pervasive healthcare applications.

Sensor networks deployed in health smart environments are connected together to exchange data through different technologies and protocols such as ad-hoc networks and the Internet. The Internet is adopted as an available solution for remote communication that facilitates access to data from anywhere and at anytime. Integrated adhoc networks with various telecommunication technologies complement the sensor and communication infrastructure. Sensors are connected and communicate either using wired or wireless protocols and technologies.

One of the most commonly used protocols in the wired communication of HSH is the X10 protocol 22]. X10 provides an easy way to transmit data over the already existing electrical wires of the home. It allows for a remote control of devices plugged into electrical power lines. X10 provides a small data rate $(20 \mathrm{bit} / \mathrm{s})$ and an unreliable connection due to electrical noise. X10 devices may interpret electronic interference as reliable information and command and react accordingly. Additionally, useful X10 data may be considered as noise in filtering [48].

Nowadays, various wireless communication technologies are employed to integrate health and medical applications to improve health services. Several wireless communication technologies are available to transfer internal and external data across sensors, base stations and gateways in personal area and body area networks. The most popular technologies are short-range wireless protocols such as Zigbee, Bluetooth, WiFi, and recently Bluetooth Low Energy [49]. These technologies are used together with largescale wireless networks such as $3 \mathrm{G} / 4 \mathrm{G}$ to provide advanced health smart home applications and services. Other wireless technologies are selected for specific applications such as the identification and tracking of persons and objects using RFID, IrDA, and UWB.

- Zigbee technology (IEEE 802.15.4): ZigBee is a specification for high-layer communication using protocols on top of the IEEE 802.15.4 standard. It is used for wireless personal area networks (WPANs) with a short-range radio communication. ZigBee was developed by the ZigBee Alliance for cost-effective, energy-efficient and wirelessly networked monitoring. It is suitable for e-health applications that require low-cost, very low-power, and long battery life. This technology is simpler and less expensive than other WPANs like Bluetooth. ZigBee can operate with data rates ranging from $20 \mathrm{Kbps}$ to $250 \mathrm{Kbps}$. It supports three kinds of topologies: mesh, star and cluster tree. Devices can form a mesh network con- 
Table 3: Comparison of Zigbee, WiFi, Bluetooth, and Bluetooth LE technologies.

\begin{tabular}{|c|c|c|c|c|}
\hline & Zigbee & Wi-Fi & Bluetooth & Bluetooth LE \\
\hline Standard & IEEE 802.15 .4 & IEEE 802.11 & IEEE 802.15 .1 & IEEE 802.15.1 \\
\hline Transmission frequency & $868 \mathrm{Mhz}, 915 \mathrm{Mhz}$, and $2.4 \mathrm{Ghz}$ & 2.4 and $5 \mathrm{Ghz}$ & $2.4 \mathrm{Ghz}$ & $2.4 \mathrm{Ghz}$ \\
\hline Topology & Mesh, star, and cluster tree & Star and point-to-point & Piconet & Piconet \\
\hline Data rate & 20,40 and $250 \mathrm{Kbs}$ & 11 and 54 Mbs & 1 to $3 \mathrm{Mbs}$ & $1 \mathrm{Mbs}$ \\
\hline Range (metre) & 10 to 100 & 100 & 10 & 10 \\
\hline Power consumption & Very low & High & Medium & Very low \\
\hline Cost & Low & High & Hight & Low \\
\hline Battrey life & Months to years & Hours & Days & Months to years \\
\hline Network nodes & 65000 & 32 & 8 & Implementation dependent \\
\hline Security & 128-bit AES & SSID Authentication & $64-128$ bit & 128-bit AES \\
\hline Benefits & Low power and low cost, reliability, scalability & Speed, flexibility & Convenience & Low cost and low power \\
\hline
\end{tabular}

necting several devices together and providing multihop routing. Three devices are used: the coordinator (the PAN coordinator), the router which is a full function device (FDD) and may act as a coordinator if needed, and the end device such as sensors and actuators.

- WiFi technology (IEEE 802.11): Wireless fidelity (WiFi) is a popular wireless communication and data transfer technology. It is based on the IEEE 802.11 series of standards used for wireless communications in a local area network (LAN). The high transmission speed is the main advantage of WiFi. The network supports star and point-to-point topologies where devices are interoperable. The WiFi coverage can include several electronic devices, which are able to connect to the local network or the Internet through a wireless network access point (AP) with an average distance of 100 metres and broadband speeds of up to $54 \mathrm{Mbps}$ depending to the IEEE standard. The disadvantage of this technology is the relatively large power consumption. WiFi represents a good candidate for sensors and devices which are deployed in $\mathrm{HSH}$ to ensure continuous monitoring.

- Bluetooth technology (IEEE 802.15.1): Bluetooth (BT) is designed for short-range wireless communications. BT is an open wireless communication technology based on the IEEE 802.15.1 standard. It is used for connecting a variety of devices for data and voice transmissions in WPAN. The number of BT devices can range from two up to eight in a shortrange network topology known as piconet. A piconet is a WPAN formed by a BT device serving as master in the piconet, while all the other synchronized devices are referred to as slaves.

- Bluetooth Low Energy technology (BLE): BLE is an emerging wireless local area network technology which provides ultra-low power consumption and cost. It facilitates the interoperability among portable consumer devices. BLE devices are designed to be thin for power saving. It reduces costs by supporting various battery life options ranging from months to years
[50. BLE is selected as a low-power solution to control and monitor applications in areas such as healthcare and smart energy. It represents an efficient technology for transferring very short data packets and short connections with minimal delay (latency) [51].

The correct selection and integration of communication technologies is still an open issue and needs to be addressed in the context of health smart home applications. Basically, energy consumption, reliability, interoperability, low cost, communication rate and distance are the main required aspects in the overall ecosystem for health monitoring, healthcare, and welfare services. Table 3 compares the features of various wireless communication technologies which are widely used in HSH.

\subsection{End-user Applications and Services}

Providing a context-aware service is the most complex part of HSH. The two main actors directly involved in $\mathrm{HSH}$ applications are the monitored subjects (e.g. patients, the elderly, and the disabled) and the healthcare provider (e.g. doctors, nurses, and volunteers within network coverage). End-user applications depend on the interaction between these two categories of actors. Provided functionalities are influenced by the subject's context and the services available to them. The processing system plays a major role in such context-aware applications, where collected data must be relevant in order to be appropriately managed and interpreted. Required actions are triggered to provide proper and personalized services based on the context. In general, context-aware applications have features that have been categorised in several studies 52] [15] 53]. In 52], Pascoe proposed a taxonomy of context-aware features which includes contextual sensing (detect and present the contextual information), contextual adaptation (the ability to automatically execute a service based on the context), contextual discovery (locate resources that are relevant to the user's context) and contextual augmentation (associate an annotation for the subject's context together with the raw sensor data). Similar to [52], authors in [15] identified three main features for a context-aware application, i.e. the presentation of information and services, the automatic execution of services, and the tagging of information with the context. 
Authors in 53] clarified the usefulness of utilizing contextual information for services or applications as a final step in context-aware systems. The context-aware features are personalization, to provide needed contents and information; suggestion, to provide recommendations for the subject's behaviour; configuration, to automatically set up devices; and user interface to optimize and adapt the interface based on the subject's context.

In the processing system, several reasoning approaches and machine learning algorithms are used to understand the subject's context and determine the required services. The application implements a user interface, provides alerts and controls the sensors. The user interface is usually used to display information on the subject's health status, vital signs, behaviour changes, etc. Alert mechanisms are used in emergency situations and are responsible for defining alert messages and selecting the network and services provider. Furthermore, the context-aware application motivates the sensor system based on the user's context. The objective is to provide an efficient monitoring system that optimizes system resources by controlling, for instance, sensing durations, frequencies, and data amounts.

In context-aware systems used in HSH, most of the features and requirements discussed up to this point are relevant, along with other considerations such as scalability, reliability, privacy and security. The scalability of healthcare applications requires reasoning and inference functionalities. The work in [54] classified the issues of reliability in healthcare monitoring into three categories of reliability: data measurement, data communication, and data analysis. Security and privacy are also of primary importance in health monitoring because of the nature and the sensitivity of the personal data that is processed [55] [56].

\section{The Architecture of Context-Aware Health Sys- tems}

In order to achieve a pervasive healthcare system for independent living [57], a context-aware monitoring system should be able to observe, interpret and reason regarding the subject's conditions including behavioural, physiological, and environmental information. In a smart home environment, the system should be able to perform actions and provide feedback to the subject according to the results of its reasoning process.

Context-aware healthcare systems learn the subject's normal behaviour and understand the special conditions of the subject and their environment. These systems help to identify unusual patterns of daily activity and make accurate interpretations of the situation to make correct decisions. Fig. 4 shows the general structure of a contextaware health monitoring system. The high-level applications are provided with significant information. We identify three phases: data acquisition, processing, and analysis.

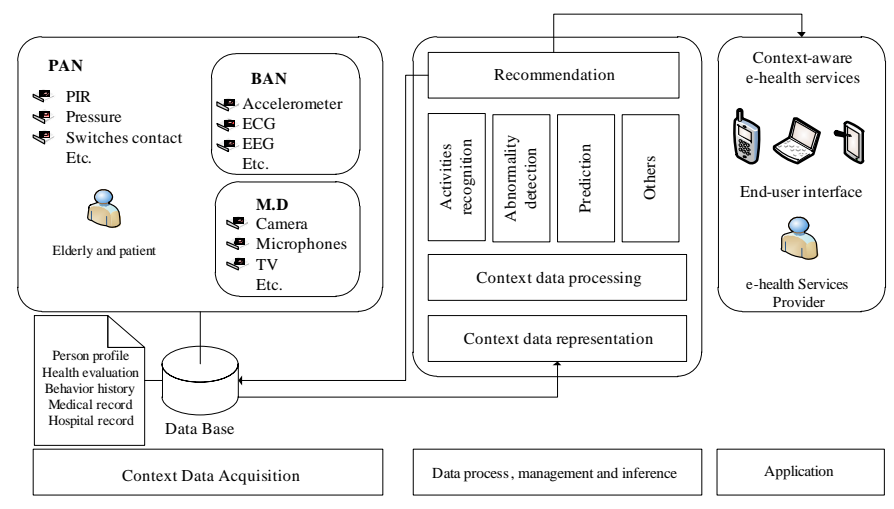

Figure 4: The overall architecture of context-aware monitoring systems in HSH.

The architecture of health computing systems is defined by the types of system components it possesses and their interactive functions. This kind of architecture is strongly influenced by the computational capabilities of its components (see Section 3 and Fig. 2). As mentioned previously, the processing stage plays a major role; it identifies the subject's context and provides appropriate services. Note that most of the computational requirements of health monitoring systems are related to the achievement of the processing functionalities. Thus, the architecture of HSH is basically based on its processing system.

\subsection{Architecture Style}

Broadly speaking, there are two main architectural styles of context-aware health systems in HSH: centralized architectures and distributed architectures.

Centralized architectures: in a centralized architecture, a central processing device (called a centralized context server) is responsible for all system functionality. Its main functions are: the collection of data from sensors, data processing while executing various analysis with algorithms and reasoning techniques, providing services and interfacing with the local health system and the outside world.

The majority of existing health monitoring systems belong to the centralized architecture category [58]. The centralized context server must have significant computational power and must be able to collect, process, and store data. In centralized architectures, communications are relatively easier than in distributed architectures. However, the drawback of such architectures is the failure tolerance when the central server crashes or when network congestion occurs.

Distributed architectures: in distributed systems, each component works independently and manages its own context information. Every component communicates with each other over the network, to transfer and share information. A distributed architecture benefits from the computational capability of each device within the smart environment. Failed or less important components and devices 
impact the context-aware operations. The distributed system provides the opportunity to increase the reliability, availability, and performance of applications. The integration of existing system components, running on different platforms, is the most important feature of a distributed system. The most significant disadvantage of these systems is the complicated design and maintenance of components. Several failures have the potential to occur in distribution systems, such as mobile devices lacking resources and computational power.

The distributed architecture model uses multi-agent systems (MAS) [59] or service oriented architecture (SOA) paradigms 60] to design and develop health monitoring applications. Mobile agents and multi-agent systems are used to improve the home care environment in which autonomous agents are used for various purposes and collaborate with each other by sharing their knowledge. MAS facilitates the design and establishment of a distributed intelligence to perform complex tasks. Multi-agent systems are used to solve the issues that could not be tackled using the centralized approach or when using agents independently. Intelligent multi-agent systems are used to improve the healthcare and assistance of monitored subjects. For instance, applications can provide a patient localization and management system 61], for tracking locations and detecting abnormal behaviour along with a group-based collaboration [46], and providing support for comfortable, healthy and independent living 62 .

The service oriented architecture is a model for reorganizing software applications and infrastructures into a set of interacting services. Service-oriented computing (SOC) is the computing paradigm, which relies on SOA to use services as fundamental elements for developing applications 63. Several health monitoring applications were developed based on SOA. A health service platform proposed for smart homes was presented in 64. The platform integrates various devices and services and provides interoperability, modularity, and reusability of service components. Using SOA, some works provided an ambient assisted living 65] 66] and integrated a vital sign monitoring and telemedicine platform [67]. A framework of health services, including bio-medical monitoring, alerts, and communication with a coordination center was presented in [68]. The work of [69] presented a pervasive health system, which enables self-management of chronic patients during their everyday activities.

\subsection{Middleware}

One of the central challenges of e-health applications in $\mathrm{HSH}$ is heterogeneity. It is caused by the use of a variety of sensors and devices with different standards and modalities. Middleware represents a solution used to integrate several sensors and facilitate their interaction with the monitoring system. Middleware is defined as a layer between the networked operating system and the application. It represents a reusable solution for the issues related to heterogeneity, interoperability, security, and dependability
[70]. Middleware plays a significant role for HMS to create a common platform that helps manage the complexity and heterogeneity of health sensor networks inherent in distributed systems. It provides an abstraction between software applications and the sensor hardware in order to simplify the application design. Several studies have focused on the design and development of middleware solutions for health environments [65] 71], while others have focused on the challenges in developing such middleware [70] 72]. For example, the authors of [72] identified the challenges in developing middleware solutions for sensor networks mainly in terms of abstraction support, data fusion, resource constraints, dynamic topology, application knowledge, adaptability, and scalability.

A cloud-oriented context-aware middleware for ambient assisted living (CoCaMAAL) was proposed in 65. The system comprises five main cloud-oriented components: ambient assisted living (AAL) systems, a context aggregator and provider (CAP) cloud, a service providers cloud, a context-aware middleware (CaM) cloud, and a context data visualization cloud. The motivation of CoCaMAAL was that biomedical sensors lack the processing power to perform key monitoring operations, data aggregation, data transmission, and computation. The objective was to support body sensor networks in AAL. CoCaMAAL uses cloud computing to provide real-time services for assisted living. Basically, CoCaMAAL is built upon SOA to perform the context modelling for raw data, context management, and service handling (mapping, distribution, and discovery). The Context-Aware Middleware for Pervasive Elderly Homecare (CAMPH) [71], provides several system-level services including context operations (acquisition, storage, reasoning) and service organization and discovery based on context query processing. CAMPH was designed to facilitate the development and deployment of various homecare applications. The overall architecture consists of four logical layers: the physical space layer, the context data management layer, the service management layer, and the application layer. In 73], the authors designed a middleware to facilitate the development of a ubiquitous computing system for healthcare through using autonomous agents. These agents can represent users, resources, or complex system functionality. The context aware solution for the Internet of Things (CA4IOT) presented in [74], is a middleware architecture for IoT developed to automate the task of selecting sensors according to the problems/tasks at hand. The middleware solution focuses on understanding the user requirements and selects the most suitable sensor for providing relevant information based on the users' context. The middleware architecture consists of four layers: the sensor data acquisition layer, the context and semantic discovery layer, the processing and reasoning layer, data, semantics, and the context dissemination layer.

Overall, middleware in HSH can be either proprietary and dedicated to a specific application or general and based on open standards. Based on the literature, we observed 
that there is a tendency to use open standards in the implementation of software and services for interfacing the HSH with the outside world. The most pertinent open standards used to construct and develop middleware in $\mathrm{HSH}$ are from the Open Services Gateway initiative (OSGi) and from Web services standards. In order to provide intelligent health services for subjects, there is a need to develop an integrated platform for combining and managing several heterogeneous sensors and devices. The platform should provide a variety of health assistive services such as health monitoring, diagnosis, evaluations, and reminders. Additionally, the platform should be flexible so that new application services can be added to connect them with the outside world.

- The Open Service Gateway initiative (OSGi) is an open service-oriented framework that significantly simplifies software lifecycle management. It allows the implementation of a software solution as an independent service. Originally, OSGi was proposed for deploying services from different providers in order to be used as service gateways. OSGi is an open specification that integrates and manages various devices in a local network. It provides dynamic discovery and collaboration of devices and services from different sources using standard interfaces.

In addition, the OSGi support of connectivity with the outside world promotes remote control management. The uniqueness of OSGi is that it provides for dynamic adjustment where the reusable software components, named bundles, can be manipulated (installed, updated, removed, etc.) independently of the device operations. In general, the OSGi framework can be adopted as a typical solution that allows managing and integrating different devices and hardware components in the network through a unified service-oriented approach. This property helps to fully comply with the need for a dynamic and reconfigurable approach to remote health monitoring [75].

- Web Services technology (WS) is a set of open standards and protocols which provide common methods ensuring interaction and communication among applications, services, and devices running on disparate platforms. WS allows for the access to software components through standard Web protocols. The W3C standards define a Web service as a software system designed to support interoperable machine-tomachine interaction over a network. WS provides a standard means of interoperation between different software applications running on different and independent platforms and frameworks. Extensible Markup Language (XML) is used to code/decode data and send/receive messages between clients and servers using Simple Object Access Protocol (SOAP) conveyed by HTTP. Web services can be easily ac- cessed over the Internet by using XML descriptions with three major standard technologies: SOAP, Web Services Description Language (WSDL) and Universal Description Discovery Integration (UDDI). Thus, WS allows for easy aggregation of descriptions, which can be accessed by several applications running on different environments. Hence, proposed systems benefit from improved interoperability and scalability for managing their heterogeneous systems and devices 76 .

Several health monitoring systems have been designed and developed based on the OSGi framework and Web Services. A wireless health monitoring system was proposed in [5] to measure, process, and transmit patient's physiological data to a centralized remote platform for data collection. The data referred to information such as the glucose level in the blood, the arterial saturation of oxygen, the blood pressure, the heart rate, and weight of the subject. The system was built based on OSGi to manage the biomedical devices and define a service-oriented architecture. In this system, users can interact with Television by using a multimedia home platform (MHP) middleware to browse personal physiological data. The AAL framework presented in [77] provides for remote monitoring, emergency detection, activity logging and personal notifications dispatching services. The proposed serviceoriented architecture is based on a heterogeneous sensor network composed of WSN nodes and personal mobile devices. The middleware is integrated into an OSGi framework that processes the collected data in order to provide context-aware services and enable network control. In [78], a ubiquitous home healthcare environment architecture is presented. This architecture is composed of four main building blocks, which are the healthcare gateway (based on OSGi), the Zigbee portable medical devices, the remote management server, and the data center. Message formats were defined between the Zigbee coordinator and the Zigbee portable medical device and devices were dynamically converted into UPnP devices. Since the software in the healthcare gateway was managed by OSGi, it can be remotely configured, managed, monitored, and diagnosed. Authors in [79] proposed an integrated OSGi-based service platform to aggregate diverse safety and health related services and to provide personalized assistive support for the elderly. By leveraging the component-based architecture of the OSGI service framework, the platform aimed to integrate heterogeneous devices, networks and data modalities, to provide assistive services for the elderly and to promote collaboration between devices and services from various sources.

In [80], a distributed and patient-oriented telehomecare system was developed based on Web services. The objective is to support remote medical management with QoS, heterogeneous platforms and homecare across the global wide area networks. The system architecture includes five elements: environmental and home automation 
sensors, medical body sensors, a gateway, Web services technology and graphical user interfaces. The healthcare system proposed in [81] integrates OSGi with Message Oriented Middleware (MOM) and SOA. It provides a flexible telemedicine platform where the system is designed to ensure interoperability of applications and services over a variety of health network devices. MOM is used to perform systems integration and reliable data transmission. With publication and subscription abilities, new applications can be added without interfering with the other components of the system. Services were designed as Web services using XML and open standards. SOA was used as the key concept to define reusable components and service cooperation mechanisms in a loosely-coupled manner to create a healthcare server. A service oriented architecture for independent life and for supporting health was proposed in 68] using OSGi and Web Services. The architecture provides services such as biomedical monitoring, alerts, and communication with a coordination center. The architecture was divided into three modules: a coordination center, a residential gateway, and a mobile gateway. The fixed gateway manages the intelligent home environment, the mobile gateway handles the intelligent personal environment, while the coordination center integrates both platforms. The coordination centre also provides an extensible communication system for the service providers.

\section{Activities, Monitoring and Machine Learning in HMS}

Context-aware HSH and assisted living systems for subjects like the elderly aim to monitor and evaluate the subject's health condition and their abilities to carry out daily activities [82]. Such systems are effective when they gain a good knowledge of the subject's daily behaviour. Indeed, the success of an intelligent HMS is measured by its ability to understand the normal behaviour of subjects, detect abnormal situations and predict future health conditions.

In reality, the subject's behaviour is highly dependent on perception, context, environment and prior knowledge [83]. Moreover, other significant factors that can affect human behaviour are the physical and mental states and changes to perceptual abilities, physical skill, and memory. Therefore, a key challenge in a smart health system is to select the appropriate methods and techniques to effectively understand complex and variable human behaviour. In this realm, research was conducted for monitoring and assessing the functional abilities of subjects and their physiological and behavioural abilities.

In this section, we address briefly the nature of human activity and behaviour. We look at health monitoring systems and the techniques and learning algorithms used by these systems.

\subsection{Taxonomy of Activities}

In this sub-section, we present a taxonomy of healthcare applications available in smart environments for elderly and dependent subjects. In the geriatric domain, the health and wellness status of individuals is measured by the so-called dependency evaluation level 82] 84]. The subject's dependency can be defined as the ability of a person to achieve elementary tasks of daily living without the help of a third party [84]. In the context of e-health services and smart home environments, the monitoring and evaluation of the subject's behaviour regarding activities of daily living (ADL) are of high concern. Evaluations help to reveal the subject's context and real needs. Hence, the system facilitates timely assistance and services, the essence of context-aware health monitoring systems.

Incorporated activities in most cases are the activities of daily living defined by Katz [85] and the instrumental activities of daily living (IADL) of Lawton [86]. The ADL category refers to the routines and basic tasks performed by subjects every day, such as eating and washing. The IADL category refers to tasks required to live in a community, examples would be meal preparation and medication use. IADL abilities are usually lost by the subject before ADL activities are lost. The majority of assisted living systems for the elderly incorporate the monitoring and detection of ADL activities, either partially or entirely.

Ambulatory activities are activities that are related to the subject's motion and posture. These kinds of activities can be divided into dynamic, static and transition activities. Dynamic activities include a set of dynamic actions such as walking and running. Static activities describe a posture such as sitting and standing. Transitional activities refer to the changes of state such as moving from sitting to standing, i.e. sit-to-stand and stand-to-sit). The ambulatory activities are often used within a motion tracking and fall detection system. Mental functions, such as memory and comprehension, are other types of important parameters that are used in health monitoring systems. These functions do not imply the achievement of an action by the subject but they can be deduced based on the overall behaviour and the subject's ability perform other activities. For instance, the ability of the subject to regularly take medication on time can indicate his memory abilities. The last important type of this monitoring community is related to physiological activities such as cardiac and brain activities. This type is used in real-time monitoring to retrieve the direct health parameters of subjects, especially those who suffer from chronic diseases.

In HMS, the types of the activities that are of high concern in healthcare and assisted living systems are summarized in Table 4. The proposed classification is the first step toward defining activities based on the sensors that are deployed and the processing techniques that are selected to identify the context of the subject. 
Table 4: A Classification of Activities in Health Monitoring Systems

\begin{tabular}{|c|c|}
\hline Туре & Activities \\
\hline $\begin{array}{l}\text { Activity of Daily } \\
\text { Living (ADL) }\end{array}$ & $\begin{array}{l}\text { Eating, Dressing, Washing, } \\
\text { Grooming (brushing teeth, wash- } \\
\text { ing hand/face and hair dry), } \\
\text { Toileting, Sleeping }\end{array}$ \\
\hline $\begin{array}{ll}\text { Instrumental } & \text { Activ- } \\
\text { ity of Daily } & \text { Living } \\
\text { (IADL) } & \\
\end{array}$ & $\begin{array}{l}\text { Meal preparation, Housekeeping, } \\
\text { Laundry, Telephone, Medication } \\
\text { use }\end{array}$ \\
\hline $\begin{array}{l}\text { Ambulatory Activity } \\
\text { (AMA) }\end{array}$ & $\begin{array}{l}\text { Dynamic activities } \text { (walking } \\
\text { inside-outside and up-down, run- } \\
\text { ning, and jogging). Stationary } \\
\text { activities (standing, sitting and } \\
\text { lying). Transitional activities } \\
\text { (e.g sit-to-stand, stand-to-sit, } \\
\text { stand-to-walk etc) }\end{array}$ \\
\hline $\begin{array}{l}\text { Mental } \\
\text { (MF) }\end{array}$ & $\begin{array}{l}\text { Memory, Comprehension, Judg- } \\
\text { ment, Orientation }\end{array}$ \\
\hline $\begin{array}{l}\text { Physiological Activ- } \\
\text { ity (PHA) }\end{array}$ & $\begin{array}{l}\text { Cardiac activity, Brain activity, } \\
\text { Muscle activity, etc. }\end{array}$ \\
\hline
\end{tabular}

\subsection{Activity Conceptualization}

The context-aware health monitoring applications which are developed to detect and evaluate human behaviour need a clear description of the nature of human activities. In this regard, we present a general conceptualization of activities.

Physical human behaviours can be distinguished by either actions or activities. Actions and activities are used interchangeably to denote human behaviour with different complexity levels. On the one hand, an action usually refers to a simple event that is executed by a single person and typically lasting for a short time. For instance, opening a door, moving dishes, turn on light, etc. On the other hand, an activity usually refers to a more complex behaviour consisting of a sequence of actions that are performed either by a single or multiple persons who are interacting with each other in a constrained manner. Activities (e.g. meal preparation) last longer than actions and can be seen from three different perspectives: a sequence of actions, interleaved activities or concurrent activities. Examples, the sleeping activity can include the following sequence: open the door, lie on the bed, and turn off the light. For the interleaved view, the subject can make meal preparation to toileting then meal preparation. Finally, the subject can execute activities simultaneously such as watching $T V$ and eating .

In a smart home environment, context information such as location, posture, time, frequency and related objects are also critical in monitoring activities. Such information can be used to characterize an activity and to learn human behaviour. A location refers to a specific place where a given activity is often performed. For instance, taking a shower takes place in the bathroom. Human posture is almost always associated with the subject's location and with performed actions or activities. Some main postures like sitting and lying are used to characterize other activities such as resting, waking, and sleeping. The time consideration, which includes the starting and end time (or duration), is another key characteristic for describing activities. For instance, lengthy sleeping usually occurs in the evening and in a semi-regular timespan within a normal daily routine. In addition, in normal life routines, some activities are performed with a known range of frequency (or repeatability); taking a shower normally occurs once or twice a day. Finally, objects are usually used in monitoring human activities where an object can indicate the relevant activity being done. For instance, using a broom as the object can indicate the housekeeping IADL activity. Other contextual dimensions can be considered to detect the performed activities such as temperature and humidity. These characteristics are useful in monitoring and recognizing activities, learning normal human behaviour and detecting abnormalities, and to evaluate and predict the status of health.

\subsection{Monitoring Functionalities}

We identify three major functionalities offered by health monitoring systems: behaviour and activities recognition [87] 88] [89], abnormality detection 90] 91] and behaviour and health prediction [30] 92] 93].

\subsubsection{Behaviour and Activity Recognition}

Activity recognition is a well-known process in HMS and is one of the most promising research topics for contextaware computing and ambient assisted living. It aims to detect or recognize human activity and behaviour patterns in real-life settings [94]. Thus, it becomes an essential element for many health applications, such as automating human behaviour monitoring for the elderly people.

As mentioned previously, human's behaviour in daily activity is complex and highly diverse. So too is the challenge of monitoring such activity. These challenges are outlined in [94] and are: (a) recognizing concurrent activities: performing several activities at the same time, (b) recognizing interleaved activities: activities that are overlapped with others in real life, (c) ambiguity of interpretation: similar actions may be interpreted differently based on the context, and (d) support of multiple residents: recognize the activities performed in parallel by the occupants in a group.

Many advances have occurred in human behaviour recognition. In the literature, different approaches, methods, and algorithms have been proposed and improved upon. Generally, the activity recognition field comprises many different topics such as activity modelling, behaviour, and environmental monitoring, data processing and pattern recognition. Hence, in practice, the recognition of activities [95] can be roughly characterized by the following four basic tasks.

1. the use of appropriate sensors in the subject's environment to capture environmental changes and to monitor and capture the behaviour. 
2. the collection and processing of perceived information through aggregation and fusion of data to extract high-level contextual abstractions.

3. the design of computational activity models in a way that allows software systems and agents to conduct reasoning and manipulation.

4. the design of methods and algorithms to efficiently infer activity from sensor data.

\subsubsection{Detection of Behaviour Abnormalities}

Detecting anomalies in human behaviour, when performing daily activities and monitoring health conditions is another challenging task. Anomaly detection, which is also known as outlier detection, is widely studied and has been applied to many application domains. Generally, the definition of anomaly detection refers to finding a pattern in data that does not conform to normal or expected behaviour. The non-conforming pattern is often referred to as an anomaly or an outlier [96]. Anomaly detection is a complex process that requires learning normal behaviour patterns and then setting assumptions to distinguish between normal and anomalous in behaviour. This process is heavily influenced by methods that get applied for performing detection, choosing sensors and extracting features. The authors of [97] have identified the general challenges facing abnormality detection processes. We now summarize the main challenges regarding human behaviour and health monitoring systems.

1. Defining normal behaviour is difficult. The boundary between normal and abnormal behaviour is often blurred. Some borderline abnormalities can actually be considered normal and vice versa.

2. By its very nature, human behaviour is irregular and constantly changing. Thus, normal behaviour today may not appear normal in the future

3. Anomalies varying depending on the subject's nature and situation. For instance, some behaviour and vital signs could be abnormal for one subject and not so for another.

4. The use of appropriate methods for detecting anomalies requires training, which can be cause major issues.

5. Data coming from sensors is often incomplete or contains noise, which in some case is similar to real anomalies. This makes the process more difficult. Consequently, increased processing is required to clean the data.

\subsubsection{Behaviour and Health Prediction}

As mentioned previously, interpreting sensor data leads to a better understanding of the subject's context. Knowledge of context is useful for evaluating the current state of a subjects health conditions and for detecting abnormalities on an ongoing basis. In order to meet these requirements, it is important to utilize long term situational data to predict future health conditions ahead of time and to make the necessary arrangements proactively.

Applying predictive methods is another important function of HMS, for predicting the state of a subject's future health. These predictions are achieved by using any available historical health data of the subject, including health checkups, behavioural history, medical and hospital records, etc. Applying predictive and analytical methods to such data helps to provide more specific knowledge about the subject's health. This can help in understanding when to notify caregivers if there is a high probability that the subject's health is likely to decline. Predictions are useful in automating the process of understanding the subject's normal behaviour. This, in turn, can be used to detect irregularity or any deviation in the behaviour of the subject.

\subsection{Modelling Context Data}

During the acquisition of data, contextual health information is usually categorised into multiple formats, due to its heterogeneity. A common modelling mechanism is used for transforming the data into a readable and processable format. The context modelling concept also refers to the contextual representation of knowledge in a given system. The objective of modelling techniques is to define and represent data in a unified format and to understand and share knowledge in context-aware systems within the smart space community. Several context modelling techniques are used for data representation. Based on the literature, the most popular methods used in the health domain are key-value models, markup schemes, graphical, object-oriented, logic based, and ontology-based modelling. We now briefly present the major methods used in health context representations.

Key-value modelling is the simplest method used for data representation. A list of key-value pairs is used to define the set of attributes and values. The authors of [14] used key-value pairs to model context by describing specific location information. Markup scheme modelling defines hierarchical data structures using a markup language such as XML. The markup tags (attributes and content of attributes) are used to represent and format the data. The work in [98] used the homeML schema to represent the subjects' activity in smart homes. The use of the XML-based scheme was motivated by the ability to solve data heterogeneity and to represent, store and exchange data in a flexible way. Graphical modelling is a diagrammatic representation of contextual data at the design level. This modelling uses appropriate models such as the Unified Modelling Language (UML) used in 99. and Object-Role Modelling (ORM) used in [100]. Objectoriented modelling uses the concept of objects, with class hierarchies and relationships, as a context modelling tool. It employs encapsulation, reusability, and inheritance to represent context data. The work in [101] used a general object-oriented context model to propose a context-aware system in smart environments. In this model, the context 
data is structured around a set of entities, each describes a physical or conceptual object such as a person or an activity. Contextual entities were linked to their attributes and other entities with defined relations.

In logic-based modelling, the context is represented as a set of logical facts, expressions and rules. The logic-based knowledge representation models the sensor data and then uses the logical rules to extract contextual knowledge. In 102], a formal framework was proposed to represent the contextual sensor data in a smart home based on Description Logic (DL). Ontology-based modelling represents the knowledge and contextual information using semantic technologies. The main components of ontologies are concepts, instances, and relationships that can be used to form the context data. This modelling method emerged as the most interesting method used for data representation and reasoning [103]. The authors of [104] presented an ontologybased context modelling and reasoning method for using the Ontology Web languages (OWL) to address the issues of formal context representation, knowledge sharing, and logic based context reasoning. In [105], OWL was used for ontological modelling and representation with a knowledge-driven approach for ADL. The approach establishes links between activities and contextual information through activity-based properties. We direct the reader to [20] for further reading on context modelling techniques.

\subsection{Machine Learning and Reasoning Approaches}

Raw sensor data is worthless unless it is interpreted, analyzed and understood. Therefore, a key challenge in a smart system is to identify appropriate methods and algorithms to effectively interpret sensor data (low-level) and build new abstractions (high-level) in order to understand this complex and variable data on human behaviour.

The task of context reasoning, also called inference, aims to deduce new knowledge based on available context data [106]. Context reasoning can be composed of three phases: data pre-processing, data fusion, and context inference. Due to the nature of the sensors and network communications, sensor data may appear inaccurate or incomplete. The phase of pre-processing aims to facilitate further processing by dealing with missing values, cleaning collected data and removing outliers. These tasks have been the focus of the sensor network and data mining communities for a number of years. In health applications, the full perspective of the subject's context could not be reached by a single sensor. Data fusion is a critical task for integrating data from multiple sensors to produce a more complete and accurate vision. Context inference represents the most challenging task compared to pre-processing and data fusion. It aims to generate the real context of the subject based on the sensor data through several learning algorithms and reasoning mechanisms.

The range of methods and algorithms applied to detect human context and behaviour is extensive and varies regarding several concepts. The adoption of these processing methods has a decisive impact on the accuracy of the final results. Thus, it is fundamental to the success of the whole system. We now briefly introduce methods and techniques that have been widely used to recognize human behaviours, detect normal and abnormal situations and to predict future health conditions. We broadly classify techniques into three categories: probabilistic and statistical techniques, computational intelligence techniques, and knowledge-driven techniques. Table 5 demonstrates the uses of these methods and techniques.

\subsubsection{Statistical Techniques}

Statistical techniques are the most commonly used techniques for modelling human behaviour in the field of healthcare. They have been used to deal with all operations related to behaviour recognition, detection, and prediction. Broadly speaking, these methods were used to find dependence and correlations between temporal information captured by sensors and the subject's estimated behaviour.

Methods like hidden Markovian models (HMM) and Bayesian networks are probabilistic reasoning concepts derived from statistical inference processes [83]. Probabilistic methods were commonly used for modelling behaviour, due to their ability to present random variables, dependence and temporal variation between collected data 83 . HMM is a statistical technique where the system uses a Markov process with hidden parameters. It defines a number of hidden states and observations used to model a given behaviour. The hidden states represent the activities and the sensor data represents the observable output. A HMM is defined by matrices which encode possible states and probabilities of observations. State transition matrices describe the likelihood of the process moving into a new state. The HMM and its extensions such as the Couple Hidden Markov Model (CHMM) [107], Hierarchical Context Hidden Markov Model (HCHMM) 108, and Hidden Semi-Markov Model (S-HSMM) [109] were used for several processes including the recognition of daily activities [26] [10], abnormality detection [93], and behaviour prediction [11]. The main drawback in basic HMM is the lack of hierarchy in representing human behaviour [112]. This problem can be solved using the Hierarchical Hidden Markov Model (HHMM) [11].HMM has difficulties experienced in processing large low-level sensory data (i.e. temporal data coming from different time scales) [113]. Moreover, using HMMs as a time series prediction model needs a large and growing number of time sequences.

Conditional random fields ( $\mathrm{CRF}$ ) is another statistical modelling method often used in pattern recognition. It is different than the HMM model, which assumes that all observations are independent. Thus, it could potentially overlook the complex relationships between observations. CRF is an alternative probabilistic model which does not make independence assumptions between the observations and represents conditional probabilities of sequential data. CRF has been compared to HMM for activity recognition and detecting patterns in [114]. The authors found that CRF provides better classification and accuracy than 
HMM. However, CRF requires more computation of training data, especially if a large number of features are concerned.

Bayesian networks are statistical tools that provide a more general framework for modelling human behaviour. The key problem of Bayesian networks is that the exact probabilistic inference is intractable 112]. There are several examples of using Bayesian networks for behaviour modelling such as in the monitoring and detection of human activities [115] [116]. Naïve Bayes classifier (NBC) is the simplest possible probabilistic classifier and is based on Bayes' theorem to perform Bayesian inference. It has been used with promising results for activity recognition in [117]. However, the NB classifier needs large amounts of data to provide good accuracy of recognition. Moreover, it does not explicitly model any temporal information, which is usually considered important for activity recognition in smart spaces.

Unlike the study in 114, a systematic study has been conducted in [118] in order to compare the performance of three activity recognition models: NBC, HMM, and CRF model. They evaluated these models using the dataset combined from the CASAS smart home project to recognize eleven activities of daily living. The activities are personal hygiene, sleeping, bed-to-toilet, eating, cooking, working, leaving the home, entering the home, relaxing, taking medicined, and bathing. These activities were analyzed using sensor event datasets collected from seven physical testbeds. The result of the recognition accuracy using 3-fold cross-validation over the dataset is $74.87 \%$, $75.05 \%$, and $72.16 \%$ for NBC, HMM, and CRF respectively.

Gaussian Mixture Model (GMM) is another probabilistic model that was investigates in the literature to learn the normal behaviour in performing ADL activities and detecting abnormalities in a smart space. GMM was used along with a rule-based reasoning in [119] and with a visionbased reasoning in [46] in order to learn the normal behaviour and detect abnormal situations.

\subsubsection{Computational Intelligence Techniques}

Computational intelligence techniques are widely used in the literature as an alternative to statistical methods. These techniques, such as neural networks, support vector machines (SVM), data mining, decision tree and fuzzy system, were used to recognize activities, distinguish between normal and abnormal behaviour patterns, and predict human behaviour and health conditions.

Artificial Neural Networks (ANNs) have been widely used in the field of intelligent computing. They represent a mathematical tool in the artificial intelligence field that provides robust self-learning ability for data classifications. ANNs have been employed in pervasive healthcare monitoring systems. An ANNs-based structural health monitoring scheme with wireless sensor networks was proposed in [120]. The simulation results show higher accuracy in data processing with NN compared to other classification algorithms including Support Vector Machine, Decision Tree, and Logistic Regression. ANNs were also used to detect abnormal patterns in vital signs with BSN [121], provide individualized embedded diagnosis and decision support for remote health monitoring [122], and recognize ambulation activities along with transitions among the activities [123]. A temporal ANN-based embedded agent was proposed in [124]. The agent is able to work on realtime data from unobtrusive low-level sensors and actuators. The work recognized behavioural patterns of ADL activities and used these patterns to detect abnormalities in the temporal order in which activities take place. The predictive modelling engine proposed in 92] mined ADL data using machine learning algorithms with ANN.

Different combinations of ANNs were used for learning the daily routine activities of subjects in smart environments. Multi-layer perceptron (MLP) neural networks were used with a single hidden layer to identify human motion [125]. The authors of [126] used MLP as the prediction technique to anticipate the subject's next movement. The authors of [127] have applied different learning algorithms to recognize the age categories and to detect possible changes in individual's health condition based on walking data patterns. The used algorithms were MLP, decision tree, support vector classifier, Naive Bayes and Bayesnet. In their experimental results, MLP gave the highest accuracy in classifying the categories. A one-pass neural network system used to detect the subject's activity was discussed in 128. The authors have introduced a new layer that helps to differentiate normal and abnormal behaviour based on the frequencies of ADL.

Supervised learning techniques such as Support Vector Machines (SVMs) were widely used for pattern recognition and classification. For example, ambulatory activities recognition and fall detection [129] 34, ADL recognition and classification [130] and learning situations 91]. In [131], SVM was used with HMM to detect abnormalities within an elderly behaviour pattern where HMM was applied to train the normal ADLs and then SVM was used to classify normal and abnormal behaviour. The authors of [132] applied SVM classifier to build a behaviour classification model and learn subjects' habits related to ADL for predicting and identifying occupant behaviour. The process enabled the prediction of home daily activities such as grooming, eating, sleeping, and having breakfast. However, the results were limited to a reduced set of activities which were achieved only in early morning.

Different data mining and machine learning techniques such as clustering and decision trees were used to classify data collected from sensors and to detect and predict human behaviour. Data mining and discovery of knowledge in large historical databases can be useful to extract hidden information for health monitoring [133]. Decision trees build a hierarchical model in which each branch from nodes to edges is a classification rule and represents a possible decision. The C4.5 algorithm is the most widely used decision tree classifier in activity recognition. In 134, five 
accelerometers were attached to hip, wrist, arm, ankle, and thigh in order to recognize twenty activities (ADL and AMA). Four different classifier structures were used and decision tree with $\mathrm{C} 4.5$ provided the best accuracy. Similarily, the work in [31] evaluated the effect of multiple sensors on the body for recognition accuracy. The authors investigated five classifiers (ANN, Decision tree, K-Nearest Neighbour classifier, Naïve Bayes classifier and SVM) to recognize five ambulatory activities using four sensors. The decision tree classifier was the best algorithm. Moreover, the authors explained that ANN, KNearest Neighbour (KNN), and SVM classifiers were computationally expensive although they provided good performance.

Clustering methods such as K-means clustering, Fuzzy C-means (FCM), Self-Organising Maps and K-Nearest Neighbour (KNN) are unsupervised techniques that can be used for modelling human behaviour, the recognition and detection of abnormality. The clustering process follows a simple and easy way to classify training data into a certain number of clusters and then use the clusters to classify testing or observed data. A human posture recognition system for video surveillance was proposed in [135] using one static camera. Training and testing stages were implemented using four different classifiers which are K-Means, Fuzzy C-Means, Multi-layer perceptron Self-Organizing Maps, and Feedforward Neural networks. The experimental results indicated that Self-Organizing Maps show the highest recognition rate.

In [136], different data mining techniques were compared to classify and predict the health of residents in a smart home. These studied techniques were KNN, SVM, MLP and Lazy Locally-Weighted Learning (LWL). The study showed that KNN outperformed other classifiers for the classification and prediction of the status of health. The FCM clustering technique was used in [137] to identify normal behaviour. The data were related to the movement and location of the elderly at home. Thresholds were set to find abnormal behaviour following the detection of data points outside the clusters. The author of [138] classified patients with heart failure using a K-mean clustering. The clustering of patients was based on contextual information, like medical conditions and socioeconomic status, used in predicting the status of health. In [90], abnormal situations like falls and fainting of the elderly were also identified using K-means clustering. The study in [139] achieved a comparison of three indoor positioning techniques based on location fingerprinting. The comparison was achieved using K-Nearest-Neighbor, probabilistic methods and neural networks and reveals that KNN reports the best overall performance for indoor positioning purpose.

Selecting one of the previously discussed techniques for modelling human behaviour depends primarily on the accuracy of its performance. According to our survey, several factors definitely affect the accuracy of results and show a disparity between reviewed studies and projects. Factors are mainly related to the type and amount of data used along with the type and number of sensors, placement of sensors, and the type and number of activities. Selected algorithms and methods may need to process a huge amount of data to learn and detect human's behaviour and context. Therefore, such methods can provide a good performance but at a high computational cost [140].

\subsubsection{Knowledge-Driven Techniques}

Knowledge-driven techniques are another approach used for modelling human behaviour. The approach makes observations about the real world, where most human activity is routine and the sequence of actions are similar to each other. In particular, these routines take place in relatively specific situations and are related by concepts including time, place, frequency, objects, etc. (see Sections 5.1 and 5.2).

In this method, activity models involve the exploitation of rich prior knowledge to develop formal logical reasoning and deduce human behaviour. Developed models are generally used for activity recognition, abnormality detection and behaviour prediction. Knowledge-driven techniques need to apply all the available knowledge of the problem domain to generate models and rules that can be used for inferring a high-level of context abstraction. The success of such an approach heavily relies upon the accuracy and , completeness of the prior knowledge. The most popular techniques used for this approach are Rule-Based, CaseBased, Fuzzy logic, and Ontology-Based Reasoning

Rule-based methods are the simplest reasoning techniques for modelling human behaviour, using a set of primitive rules. These rules enable the building of a descriptive model for human behaviour detection. This technique can be used to generate a high-level of abstraction using lowlevel context. However, complex human behaviour cannot be directly detected merely from rule-based approaches. Rule-based reasoning was used in 28], and combined with Bayesian networks in [141] for activity recognition and behaviour detection. In the Case-based reasoning (CBR) approach, contextual knowledge is deduced from previous successful solutions to similar problems (i.e. the basis of previous similar cases). Case-based reasoning has also been used in human behaviour and activity recognition [142].

Fuzzy systems were used to manage and model data uncertainty in sensor networks. A fuzzy learning and adaptation approach, called the adaptive online fuzzy inference system (AOFIS), was proposed in 143 to model the subject's behaviour by extracting fuzzy membership functions and learning fuzzy rules. The fuzzy logic applied in [144] was integrated into a recognition system for daily living activity of the elderly. The authors in 145] used fuzzy membership functions for movement detection and estimating daily living activity. The authors found that this approach was difficult in the home environment, and therefore they focused on a limited set of three activities. This does not meet the full requirements for an comprehensive remote monitoring solution. The authors of [146] 
present a comparative study using a fuzzy expert system and neuro-fuzzy system (NFS) for heart disease diagnosis. They explain that NFS is the more appropriate model to measure risk than the fuzzy expert system. Fuzzy set theory is usually used for controlling automated systems in combination with other techniques such as rule-based [147] or ontology rules [112] in order to optimize contextual reasoning. The system called Context-Aware Real-time Assistant (CARA) presented in [147] is a context-aware hybrid reasoning framework that integrates fuzzy rule-based reasoning with the case-based reasoning for pervasive healthcare. The framework was designed for home automation, continuous health monitoring and the prediction of possible risky situations. However, in spite of the context-aware reasoning, the results have been confined to limited activities in order to evaluate the system's effectiveness and efficiency in assessing the elderly.

Ontology-based reasoning methods are the most widely used techniques for building context-awareness systems in the healthcare domain. According to [148], the ontology is a suitable model and method for context representation and reasoning. It allows for sharing of knowledge in a dynamic environment, it enables efficient contextual reasoning and guarantees the interoperability of pervasive computing systems. Ontologies are based on description logic and knowledge representations. Mainly, the ontology reasoning technology is supported by two common representations of semantic Web languages: Resource Description Framework (RDF) and Web Ontology Language (OWL). For health monitoring, reasoning and decisionmaking systems based on ontologies have been proposed in several studies such as for building context-aware services for e-health systems [149] and real-time remote monitoring [150] for patients diagnosed with congestive heart failure $(\mathrm{CHF})$. Ontological reasoning combined with statistical inference (multi-class logistic regression) was presented in [151] for activity recognition. The authors of [152] introduced a context-aware infrastructure ontology to improve the recognition system in a smart home domain. This study addressed three goals. First, the design of a context-aware ontology to model the context which includes home sensors, human locations, times, and human postures. Second, object-based and location-based concepts were presented to distinguish different activities. They used Description logic (DL) rules for making human activity decisions. Third, an ontology based activity recognition system (OBAR) was developed based on two previous goals. The outputs of OBAR show recognized activity based on user's context. The work targeted thirteen activities which were sitting \& relaxing, sitting on the toilet, watching TV, working on a computer, eating or drinking, sleeping, lying down \& relaxing, washing the dishes, taking a bath, making a drink, cooking, sweeping the floor and scrubbing the floor.

The authors of [153] presented a system (called RADL) for recognizing activities of daily living to detect and monitor ADLs for the elderly in smart homes. The proposed ontology supports the semantic discovery of location, devices, and the environment in smart homes. The reasoning process discovers the activities and the appropriate service for a present situation. Similarly, a knowledge-driven approach for human activity recognition using multiple data streams was proposed in [105]. The proposed system is based on ontological modelling and semantic reasoning for activity modelling and recognition. However, in spite of the proposition of several ontologies used to optimize monitoring, there was no uniform standard that meets all requirements of a context-awareness e-health system. In addition, the ontology based reasoning is unable to find missing values or ambiguous information compared with other methods.

The approaches studied in this section 5.5, are the most popular learning methods and reasoning techniques for detecting human behaviour and extracting context in smart health environments. It is clear that each method has its strengths and weaknesses. Comparing the performance of these techniques from experimental research within a limited scope does not truly reflect the effectiveness of health monitoring system overall. There is no single technique that can be used to address the different facets and complexities of a context-aware HMS. Therefore, on reflection the best approach is to combine multiple methods which complement each other and provide a comprehensive picture of the subject's context in a smart space [93] [154]. An example of this is the context-aware model for change detection, using machine learning and statistical methods that is proposed in 93. The model uses an HMM-based approach for detecting anomalies in a sequence of daily activities. In addition, a statistical process has been used to identify the irregularity in routine behaviours (i.e. shift in daily routines) and a simple exponential smoothing (using Holt's liner trend method) was used to predict and detect changes in vital signs. The output of these processes were combined into a fuzzy rule-based model when making the final decision.

\section{Health Monitoring Systems and Healthcare Ap- plications}

In recent decades, many systems and applications have been developed for monitoring the elderly and patients in smart environments. There is no single classification for these applications, which generally aim to identify the subject's context and provide appropriate services. These applications share the objective of compensating for cognitive decline, by enhancing the subject's quality of life and state of health.

From our literature review, there are three main categories of such systems: ambient assisted living (AAL), movement tracking and fall detection (MTFD), and physiological health monitoring (PHM). Ambient assisted living applications monitor and evaluate a list of basic daily activities, such as eating and washing. They allow the subject to live independently and they provide the subject 
Table 5: Algorithms and Methods Used In Health Monitoring Systems.

\begin{tabular}{|c|c|c|c|c|}
\hline Functionalities & Methods categories & Algorithm and Techniques & Activities & References \\
\hline \multirow{12}{*}{ Behaviour Recognition } & \multirow{5}{*}{ Statistical Techniques } & Hidden Markov Models & ADL, AMA, PHA & {$[155][87][26][107][156]$} \\
\hline & & Bayesian Network & ADL, AMA & \begin{tabular}{l|l|l|}
$115]$ & $157][16][158]$ \\
\end{tabular} \\
\hline & & Naive Bayes & ADL & $1117[159$ \\
\hline & & Conditional Random Fields & ADL & $\begin{array}{lll}114] & 118 \\
\end{array}$ \\
\hline & & Multiclass Logistic Regression & AMA & [151] \\
\hline & \multirow{4}{*}{ Computational Intelligence Techniques } & Neural Networks & ADL, AMA & {$[123[124]$} \\
\hline & & Support Vector Machine & ADL, AMA & {$[130[32][33 \mid 34][35]$} \\
\hline & & Decision tree $(\mathrm{C} 4.5)$ & ADL, AMA & [31][134][160] \\
\hline & & Clustering & ADL, AMA & $161][135]$ \\
\hline & \multirow{3}{*}{ Knowledge-Driven Techniques } & Rule-based & ADL, IADL & [141] 28$]$ \\
\hline & & Fuzzy logic & ADL, AMA & (44][145][144] \\
\hline & & Ontologies & ADL, AMA & 104] 105$] 151] 152$ \\
\hline \multirow{7}{*}{ Behaviour Abnormality Detection } & \multirow{2}{*}{ Statistical Techniques } & Gaussian Mixture Model & ADL, AMA & {$[46][119]$} \\
\hline & & Hidden Markov Model & ADL, AMA & \begin{tabular}{|l|l|l|l|}
93 & 108 & 109 \\
\end{tabular} \\
\hline & \multirow{3}{*}{ Computational Intelligence Techniques } & Neural Networks & ADL, AMA, PHA & {$[125[128] 121]$} \\
\hline & & Support Vector Machine & ADL, AMA & {$[129][162]$} \\
\hline & & Clustering & ADL, AMA & $90 \mid 137$ \\
\hline & \multirow{2}{*}{ Knowledge-Driven Techniques } & Fuzzy logic & ADL & \begin{tabular}{|l|l|l|l|l|}
$163]$ \\
\end{tabular} \\
\hline & & Ontologies & PHA & {$[150]$} \\
\hline \multirow{5}{*}{ Prediction } & Statistical Techniques & Hidden Markov Models & ADL, AMA & 1111] 164$]$ [165] \\
\hline & \multirow{3}{*}{ Computational Intelligence Techniques } & Neural Networks & ADL, AMA & {$[126] 92$} \\
\hline & & Support Vector Machine & ADL & 132 \\
\hline & & Data Mining Techniques & ADL, PHA & $[139] 138][136]$ \\
\hline & Knowledge-Driven Techniques & Fuzzy logic & PHA & {$[146] 166$} \\
\hline
\end{tabular}

with appropriate services involving caregivers. Movement tracking and fall detection systems detect ambulatory activities, including dynamic activities, static postures, location tracking and accidental falls. Physiological health monitoring systems use real-time applications for monitoring and diagnosing vital signs for dependent and chronically ill subjects such as those suffering from diabetes, hypertension and cardiovascular related diseases.

In this realm, prototypes vary in many aspects, including hardware materials, software architectures, processing systems, functionalities, and services. Table 6 summarizes and evaluate some of the most significant works found in the literature per category.

\subsection{Ambient Assisted Living}

The progressive decline in physical and cognitive skills of subjects, in particular the elderly, coupled with common diseases (like Alzheimer's, dementia and diabetes) prevent subjects from performing basic activities of daily living such as eating, hearing, dressing, and washing. The ability to perform these activities can be significantly impacted by existing diseases. For instance, activities are impacted by frequent drinking due to diabetes, going to the toilet, and sleeping. Therefore, there is a real need to develop systems for ambient assisted living or AAL. AAL has grown in significance in recent years, as it provides pervasive healthcare systems designed within smart environments.

AAL integrates simple home automation (e.g. light sensors and motion detectors) with ambient intelligence technologies (e.g. sensing and communicating) resulting in a coherent system. This integration provides more safe independence in a subject's daily life. Ubiquitous AAL systems may employ standard or specific sensors embedded in objects of daily usage such as beds, sofas, or tables.
Understanding a subject's daily activities and their normal and abnormal behaviour can be achieved by observing the subject's interactions with these objects. Various systems and applications have been developed to monitor and evaluate the spatiotemporal activities of subjects in their home and to provide appropriate and timely assistive services. These systems can classify activities, recognize particular subject habits, detect their behaviour patterns, predict their health conditions, evaluate their dependency level, extract anomalous risky situations and automatically notify caregivers when necessary. Other studies focused on monitoring and assisting physically disabled elderly and disabled individuals. For instance, supporting visual and hearing impairment [167] 168], compensating for any impaired movement of smart wheelchairs [169], developing customizable speech recognition interfaces for speech impairment [170], etc.

Basically, AAL systems are designed for monitoring and evaluating subject activity and for maintaining healthcare service quality for subjects in need of special longterm care. AAL provides timely e-health services for subjects (such as patients and elderly) in their own home. As a result, AAL improves their quality of life, saves time and costs and enables subjects to be as autonomous as possible.

The work in 82 proposed an automatic framework, called Auto-Dep, to evaluate a subject's dependency. The proposed service-oriented architecture considers the activities of daily living as defined in the AGGIR (Gerontological Autonomy Iso-Resources Group) geriatric scale [82]. The system provides a context-aware monitoring and evaluation for assessing a subject's dependency level. This allows a caregiver to provide an appropriate level of monitoring and services. The authors in [82] have identified an expan- 
Table 6: Overview of Health Monitoring Applications in HMS.

\begin{tabular}{|c|c|c|c|c|c|c|c|c|c|c|c|c|}
\hline Cat. & Project & Func. & Services & Activities & Methods & $\begin{array}{c}\text { Context- } \\
\text { awareness }\end{array}$ & $\begin{array}{l}\text { Archi- } \\
\text { tecture }\end{array}$ & Sensing & $\begin{array}{l}\text { Sensing } \\
\text { Type }\end{array}$ & $\begin{array}{l}\text { Intrusi- } \\
\text { veness }\end{array}$ & Communications & Gateway \\
\hline \multirow{7}{*}{$\mathrm{AAL}^{1}$} & Auto-Dep 82 & A & $\begin{array}{ll}\text { Dependency } & \text { Evalua- } \\
\text { tion }\end{array}$ & $\begin{array}{l}\text { ADL, IADL, } \\
\text { AMA, FM (\#8) }\end{array}$ & $\begin{array}{ll}\begin{array}{l}\text { AGGIR-based } \\
\text { gorithm }\end{array} & \\
\text { gl- }\end{array}$ & High & D & Multi & PAN, BAN, MD & Low & $\begin{array}{l}\text { Bluetooth, Wi-Fi, } \\
\text { Zigbee }\end{array}$ & $\begin{array}{ll}\text { H. } & \text { Server, } \\
\text { Mob. } & \text { Device }\end{array}$ \\
\hline & CASIS [28] & A & Reminder & IADL (\#2) & $\begin{array}{l}\text { Rule-based Reason- } \\
\text { ing }\end{array}$ & Medium & D & Multi & PAN, BAN, MD & Medium & N/A & $\begin{array}{ll}\text { H. } & \text { Server, } \\
\text { Mob. } & \text { Device }\end{array}$ \\
\hline & AICO [171] & $\mathrm{R}$ & $\begin{array}{l}\text { Location-based Recog- } \\
\text { nition }\end{array}$ & $\begin{array}{ll}\text { ADL, } & \text { AMA } \\
(\# 11)\end{array}$ & Bayesian Network & High & $\mathrm{s}$ & Multi & PAN, MD & Low & IEEE 802.15 .4 & $\begin{array}{ll}\text { H. } & \text { Server, } \\
\text { Mob. } & \text { Device }\end{array}$ \\
\hline & $\begin{array}{l}\text { BADL Estimation } \\
{[145]}\end{array}$ & $\mathrm{R}$ & ADL Estimation & ADL (\#11) & Fuzzy rules & Medium & $\mathrm{s}$ & Single & BAN & Medium & Zigbee & Home Server \\
\hline & CARA [147] & $\mathrm{A}, \mathrm{P}$ & $\begin{array}{l}\text { Personalized Health- } \\
\text { care Services }\end{array}$ & $\operatorname{ADL}(\# 3)$ & $\begin{array}{l}\text { Case-based and } \\
\text { Fuzzy Rule-based } \\
\text { Reasoning }\end{array}$ & High & $\mathrm{S}$ & Multi & PAN, BAN, MD & Medium & Bluetooth & $\begin{array}{l}\text { H. Server, } \\
\text { Mob. Device }\end{array}$ \\
\hline & Wellness [172] 30] & $\mathrm{R}, \mathrm{P}$ & $\begin{array}{l}\begin{array}{l}\text { Wellness Determina- } \\
\text { tion }\end{array} \\
\end{array}$ & $\begin{array}{l}\text { ADL, } \\
(\# 6)\end{array}$ & $\begin{array}{ll}\begin{array}{l}\text { Statistical } \\
\text { tions }\end{array} & \text { Func- } \\
\end{array}$ & Low & $\mathrm{S}$ & Multi & PAN & Low & Zigbee & Home Server \\
\hline & N/A 89] & $\mathrm{R}$ & $\begin{array}{l}\text { Low-cost and Energy, } \\
\text { Acceptance }\end{array}$ & $\begin{array}{ll}\begin{array}{l}\text { ADL, } \\
(\# 9)\end{array} & \text { IADL } \\
\end{array}$ & SVM & Low & $\mathrm{s}$ & Multi & BAN & Low & USB, RF & Home Server \\
\hline \multirow{7}{*}{ MTFD $^{2}$} & ANGELAH [46] & A & $\begin{array}{l}\text { Fall Detection, Closest } \\
\text { Emergency Volunteers }\end{array}$ & AMA (\#1) & $\begin{array}{l}\text { Vision-based Rea- } \\
\text { soning, Gaussian } \\
\text { Mixture }\end{array}$ & High & D & Multi & PAN, MD & Medium & Wi-Fi & Home Server \\
\hline & ITALH 173] & A & Fall Detection & AMA (\#7) & $\begin{array}{ll}\begin{array}{l}\text { Statistical } \\
\text { tions }\end{array} & \text { Func- } \\
\end{array}$ & Medium & $\mathrm{S}$ & Multi & PAN, MD & High & $\begin{array}{l}\text { Bluetooth, Zigbee, } \\
\text { USB }\end{array}$ & $\begin{array}{ll}\text { H. } & \text { Server, } \\
\text { Mob. } & \text { Device }\end{array}$ \\
\hline & COSAR [151] & $\mathrm{R}$ & $\begin{array}{l}\text { Location-based Recog- } \\
\text { nition }\end{array}$ & AMA $(\# 10)$ & $\begin{array}{l}\text { MLR and Ontolo- } \\
\text { gies }\end{array}$ & Low & $\mathrm{s}$ & Multi & BAN & Low & Bluetooth & $\begin{array}{l}\text { Mobile } \\
\text { Device }\end{array}$ \\
\hline & HS Care 174 & A & Fall Detection & AMA (\#1) & $\begin{array}{l}\text { Vision-based Rea- } \\
\text { soning }\end{array}$ & High & D & Multi & PAN, MD & Medium & IEEE 802.15 .4 & $\begin{array}{ll}\text { H. } & \text { Server, } \\
\text { Mob. } & \text { Device }\end{array}$ \\
\hline & 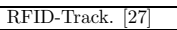 & $\mathrm{R}$ & Location Tracking & AMA (\#1) & Genetic Algorithm & Medium & $\mathrm{S}$ & Multi & PAN & Medium & RFID & Home Server \\
\hline & $\begin{array}{l}\text { RFID-Behaviour } \\
\text { [90] }\end{array}$ & A & $\begin{array}{l}\text { Movement Tracking, } \\
\text { Fall Detection }\end{array}$ & $\begin{array}{ll}\begin{array}{l}\text { AMA, } \\
(\# 2)\end{array} & \text { ADL } \\
\end{array}$ & $\begin{array}{ll}\text { K-means } & \text { Cluster- } \\
\text { ing } & \\
\end{array}$ & Low & $\mathrm{S}$ & Single & PAN & Medium & RFID & Home Server \\
\hline & MHMMR [87] & $\mathrm{R}$ & $\begin{array}{l}\text { Recognition of Ambu- } \\
\text { latory Activities }\end{array}$ & AMA (\#12) & HMM & Low & $\mathrm{s}$ & Multi & BAN & High & Bluetooth & Home Server \\
\hline \multirow{7}{*}{ PHM $^{1}$} & EMUTEM [175] & A & $\begin{array}{l}\text { Remote Health Moni- } \\
\text { toring }\end{array}$ & PHA (\#1) & Fuzzy Logic & Medium & $\mathrm{s}$ & Multi & BAN, PAN & Medium & RF, USB, ZigBee & $\begin{array}{lr}\text { H. } & \text { Server, } \\
\text { Mob. } & \text { Device }\end{array}$ \\
\hline & ETRI 39] 41] & A & $\begin{array}{l}\text { Remote Health Moni- } \\
\text { toring }\end{array}$ & PHA (\#3) & Ontologies & High & $\mathrm{s}$ & Multi & BAN, PAN & High & Bluetooth, Zigbee & $\begin{array}{l}\text { Mobile } \\
\text { Device }\end{array}$ \\
\hline & HeartToGo 121] & A & $\begin{array}{l}\text { Remote Health Moni- } \\
\text { toring }\end{array}$ & PHA (\#1) & $\begin{array}{ll}\text { Artificial } & \text { Neural } \\
\text { Network } & \\
\end{array}$ & Low & $\mathrm{s}$ & Single & BAN & Medium & Bluetooth & $\begin{array}{l}\text { Mobile } \\
\text { Device }\end{array}$ \\
\hline & SPA [133] & $\mathrm{A}$ & $\begin{array}{l}\text { Remote Health Moni- } \\
\text { toring }\end{array}$ & PHA (\#2) & $\begin{array}{l}\text { Data Mining, } \\
\text { Time-Series Rules }\end{array}$ & Medium & $\mathrm{s}$ & Multi & BAN, PAN & Medium & Bluetooth, WLAN & $\begin{array}{l}\text { Mobile } \\
\text { Device }\end{array}$ \\
\hline & Dongle [176] & A & $\begin{array}{l}\text { Remote Health Moni- } \\
\text { toring }\end{array}$ & PHA (\#2) & $\begin{array}{l}\text { Statistical Methods } \\
\text { (QRS) }\end{array}$ & Medium & $\mathrm{s}$ & Single & BAN & Medium & $\begin{array}{l}\text { IEEE 802.15.4, Cel- } \\
\text { lular }\end{array}$ & $\begin{array}{ll}\text { H. } & \text { Server, } \\
\text { Mob. } & \text { Device }\end{array}$ \\
\hline & AlarmNet [22] & A & $\begin{array}{l}\text { Remote Health Moni- } \\
\text { toring }\end{array}$ & PHA & $\begin{array}{l}\text { Statistical Methods } \\
\text { (CAR) }\end{array}$ & High & D & Multi & BAN, PAN & High & IEEE $802.15 .4, \mathrm{X} 10$ & $\begin{array}{l}\text { Mobile } \\
\text { Device }\end{array}$ \\
\hline & N/A 177. & A & $\begin{array}{l}\text { Remote Health Moni- } \\
\text { toring }\end{array}$ & PHA, ADL & $\begin{array}{ll}\text { Statistical } & \text { Func- } \\
\text { tions } & \end{array}$ & Medium & $\mathrm{s}$ & Multi & PAN, BAN & Medium & Wi-Fi & $\begin{array}{l}\text { H. Server, } \\
\text { Mob. Device }\end{array}$ \\
\hline
\end{tabular}

sive set of heterogeneous sensors and equipment such as GPS, single inertial sensors, ultrasonic water flow meters, sound detectors, flushes and light switches, door fridge, hob and door sensors, IP cameras and TVs for monitoring various ADL activities. The objective was to cover all the activities considered in the AGGIR model such as moving, eliminating, eating, dressing, washing. A Cilia model built upon OSGi was used to integrate raw sensor data, amalgamated from using various communication interfaces like Bluetooth, WiFi, Zigbee, and proprietary norms. Context data was represented using the RDF language and then run through an algorithm to evaluate the overall subject. The system automatically fills in the subject profile, aggregates any new evaluation results of the subject and links these to the subject's profile with specific dates. Dates were used to process various evaluations and detect any potential changes or decline in the subject. The proposed system can highlight any changes related to the dependency level of the subject, to receive appropriate help and assistance.

The authors of 28] proposed a multi-agent service framework called the Context-Aware Service Integration System (CASIS). The framework integrates appliances and sensors using Web services to provide external support and OSGi gateways to provide support at home. It allows reminders to be set for taking health measurements, meals, and medication. The work explored how the technology can help to enhance the quality of care by providing context-aware services and healthcare services. The environment is equipped with: (1) smart floor pressure sensors where an analog-to-digital converter (ADC) senses pressure changes and translates them into weights. Then, using probabilistic data association and LeZi-Update 28], the system analyzes the data to estimate the subject's location ; (2) a smart table which contains two layers of a RFID reader/antenna and a weighing sensor. These sensors were used to identify tabletop objects and their locations ; (3) a smart chair embedded with bio-sensors to detect health levels; (4) other devices like cameras, speakers, telephones and TVs. On the CASIS platform, the heterogeneous devices and components packaged Web services are used to communicate with each other and interact with external services. Each device is connected with a specific agent through device dependent APIs (Application Programming Interface) or network sockets. The data is then transformed into an RDF/XML format. CASIS provides context-dependent services using a rule-based reasoning approach and an inference agent. In CASIS, the context event broker implements the publish/subscribe mode, passes the outcome of the inference to the device agents then issues these commands to devices. The OSGI home gateway is responsible for managing the environment and serves as a portal for the outside connection. The proposal of 28] focused on the welfare of the elderly subject's lifestyle through modest context-aware information services using an intelligent telephone and a reminder 
system. The targeted activities of the elderly subject's daily life were limited to location, mealtime and taking medications.

Another location-aware activity recognition approach, based on ambient-intelligence compliant objects (AICOs), was used in 171]. AICOs used a Bayesian-Network-based fusion engine to classify human activities of daily living and to identify how many activities can be detected simultaneously. Basically, the system used unobtrusive wireless sensors, which were seamlessly integrated into AICOs. OSGi was used to integrate a variety of wireless sensor networks. Several objects were prototyped to facilitate interaction with the subject. In order to collect data naturally, objects were integrated into home objects used by the subject, rather than directly on the subject themselves. The project identified an activity map of human living and included a floor object to identify the subject's location, as well a power object to measure the usage of electronic devices. However, the list of sensors in the living environment are only applicable to a single subject. The solution did not consider a number of important and life-dependent ADL that affect the subject's abilities and dependency level.

The system in 145] proposed to estimate basic activities of daily living (BADL Estimation) using a motion recognition method based on $3 \mathrm{D}$ acceleration sensors and ZigBee networks. The system is composed of accelerometer sensor tags, access points, a coordinator and a server. The two accelerometer sensor tags are attached to the subject's body (hand and waist) and send data to the server via the access point or the coordinator. The signal strength indication (RSSI) of the sensor was used to detect the position of the device by calculating the distance between the sensor devices and the access point (or coordinator). The server estimated motion based on acceleration data. It used fuzzy inference and area recognition (based on RSSI) and selected the fuzzy membership function and the rules according to the recognised area. Eleven activities and events were selected from ADLs using an estimation based on the sensed data and the area recognition information. The authors underlined the complexity of estimating the activities; hence they limited the scope to only three activities in the living area.

The Context Aware Real-time Assistant (CARA) framework was proposed in [147]. CARA is a context-aware hybrid reasoning framework that integrates fuzzy rule-based reasoning with case-based reasoning (CBR) for pervasive healthcare in smart home environments. The proposed reasoning framework processes information uncertainty and uses context to provide: (1) a continuous contextualization of the subject's physical state, (2) a prediction of risky situations, (3) notifications of life-threatening emergency situations, and (4) home automation or subject prompting within a smart home environment. Particularly, CBR addressed the problems of incomplete data and was used to detect conditional anomalies for home automation. Fuzzy rule-based reasoning handled uncertainty and vagueness of data and achieved query sensitive case retrieval and case adaptation. Wearable and environmental sensors were used to collect raw numeric data. Real-time vital signs of the subject collected by wearable sensors (BioHarness sensors) included several biomedical parameters like heart rate frequency, pulse oxygen levels, systolic and diastolic blood pressure, body temperature, and respiration rates. It simulated the environment by sensing time, space, temperature, and levels of light, noise and humidity, as well as the status of TVs, cookers, phones, heaters and windows. All measured data were sent to a gateway via a Bluetooth connection. The gateway was connected to the CARA cloud server over the Internet. The reasoning applications ran on the home gateway and on a healthcare server that belonged to a private cloud environment. In CARA, the raw data was processed by the data fusion services, which produced low-level context data and built case queries and fuzzy sets. Then, the case-based and fuzzy rule-based reasoning ran simultaneously. The case-based cycle included retrieval, reuse, revision and retention operations to perform anomaly detection and home automation. The fuzzy rule-based cycle generated a high-level abstract context data and identified the current situation as normal, abnormal or emergency. However, in spite of the proposed hybrid context-aware reasoning system, the results of the system's effectiveness and efficiency evaluation were confined to a limited set of activities.

A home monitoring system (Wellness) was developed in [172] 30] to monitor and evaluate the well-being of elderly subjects living alone. The system consists of two main functions, which are activities recognition 172 and forecasting behaviour [30] in order to determine the wellness of elderly subjects. These two functions rely on a time-series analysis of household appliance usage connected through various sensing units. Sensor units were attached to various appliances and connected to a wireless sensor network using a ZigBee module. Six electrical sensors were connected to a microwave, a toaster, a kettle, a heater, a TV and audio devices. Four force-based sensors were connected to a bed, a couch, a dining chair and a Toilet. In addition, one contact sensor was used for grooming and a temperature sensor and a humidity sensor were used to monitor the atmosphere. Along with the hardware, the software system continuously read and analyzed the collected data to the home server. Activities like sleeping, meal preparation, eating, going to the toilet, grooming and watching TV were identified based on the frequency and duration time of the use of specific appliances. Additionally, the wellness functions were checked, based on the activity of specific appliances. However, despite the system being easy to use, no part of the system provided adequate or in-depth knowledge about the elderly subject or how, if necessary, they could be reached in case of emergency. Finally, simply relying on analysis over time is not sufficient to effectively determine the subject's health or to understand complex human behaviour.

The authors of 89] proposed an activity recognition 
system for ADL detection using small, low-cost, non-intrusive, subset of specific activities (like washing dishes or brushing non-stigmatized wrist-worn sensors. In particular, they used three types of sensors namely an accelerometer, a temperature and an altimeter sensor. These sensors were embedded in a normal sports watch worn on the wrist of the elderly subject. The watch had an integrated wireless transceiver, which communicated with the computer via a USB RF access point. The system targeted the detection of nine daily activities including, walking and sleeping, and washing dishes and watching TV. In order to achieve a high accuracy performance, different classification models were compared based on MLP, RBF, and SVM. The results indicated that SVM was the most powerful classification algorithm. So, it was selected for detecting the activities of the elderly subject. Moreover, the experimental results revealed that the accelerometer was more valuable than the temperature sensor or altimeter for recognizing activities. However, some system confusion occurred between various activities, such as dressing, ironing, brushing teeth and washing dishes. For dressing, there was no clear pattern on how this activity should be performed. This has proved to be challenging especially when considering generalized decision boundaries. For the other three activities (i.e. ironing, brushing teeth and washing dishes), the system assumed that these activities had some common characteristics, which in turn confused the classification process.

Wireless sensor network (WSN) and radio frequency identification (RFID) technologies were combined in the in-home elderly care application proposed in [178]. WSN sensors were achieved using Sun SPOT sensor modules supporting temperature, humidity, motion and $\mathrm{CO}_{2}$. The sensors measure ambient parameters (the elderly and the environment) for the early providing warnings of potentially dangerous situations. RFIDs included readers, active and passive tags to provide reminders for medication and other items. However, the application proposed in this work did not reflect the requirements of the elderly healthcare considerations.

Another application for smart homes was presented in [179]. It enables elderly subjects to live longer and more independently in their own homes. The project was designed to enhance quality of life (QoL) by monitoring activities of daily living. Various sensors were used to detect and gather activities related data, which were fused using decision support algorithms to extract ADL activities as established by Katz 85. Bayesian-based methods and Hidden Markov Models were used to model the uncertainty of human behaviour. Unfortunately, the project did not address in detail the relationship with caregivers or the methods used to connect the sensors with the in-home server.

In AAL and ADL monitoring systems, it is essential to identify correctly the activities that require monitoring. In most studies, the motivation for selecting specific activities was not provided. Present studies mostly address a small number of ADL activities, while others consider a teeth). These key activities are considered independently of the subject's context and needs. We also observed modest attempts to consider the IADL (instrumental activities of daily living) category of activities. The reason for this lies, partially, in the lack of understanding which activities should be adopted in health monitoring systems. Furthermore, limitations in adaptability and the lack of flexibility in existing approaches often lead to a limited and narrow range of possible activities. In our previous investigation [84], we provide a clear understanding of context of monitored subjects. We identify a set of activities of daily living that should be monitored in e-health monitoring systems, and we show how they affect the performance of health monitoring systems.

\subsection{Movement Tracking and Fall Detection}

Mobility and accidental falls are common causes of serious damage that can lead to the loss of life. Several projects used location tracking to focus on fall and movement detection both indoors and out. In HMS, robust and immediate fall detection is important in facilitating appropriate services and medical support. The main aim of such projects is to provide alerts to caregivers in the event of a fall, and to enable remote monitoring to facilitate rapid intervention in emergency situations. Mainly, movement and fall detection systems target ambulatory activities, including dynamic, stationary and transitional activities (section 5.1), as well as location tracking and accidental falls. These systems use PSN and MD sensors and devices for data acquisition (section 3.1).

According to [180], the devices in this class of monitoring can be divided into three categories: wearable-based, ambient-based (i.e. sensor-based approach) and camerabased (i.e. vision-based approach), see section 5.3. Wearable detection approaches use sensors, such as accelerometers and gyroscopes, to detect and measure motion, location, and posture by measuring acceleration and orientation [129] 181]. Ambient detection approaches use devices such as pressure sensors and PIR for movement detection [182]. They also rely on audio and vibration data analysis [183]. The camera and vision detection approach, implemented in video tracking systems, relies on video data processing such as inactivity, shape and 3D motion for movement and fall detection 174] [46].

The ANGELAH (AssistiNG ELders At Home) framework, proposed in [46], aimed at ensuring elderly subjects' in-house safety, regarding monitoring, emergency detection, and networking solutions. Two main design principles were considered in ANGELAH: context-awareness and group-based collaboration. The middleware solution aims to integrate sensors and actuators required to monitor and guarantee the elderly subject's safety, detect potentially dangerous situations, and assemble emergency response groups. These groups are composed of volunteers and caregivers in the vicinity, willing to help in case of emergencies. A variety of actuators and sensors are 
used in the ANGELAH architecture: video cameras, RFID readers, sound sensors, and appliances such as smart door locks, microphones, and speakers. Devices are placed in a way that enables the elderly subject to interact with the system. All monitoring devices are integrated on top of the Open Service Gateway initiative (OSGi) infrastructure. They gather raw context data and communicate data to the home manager (HM). Context information from sensors is analyzed by the HM using computer vision techniques and Gaussian Mixture models to detect abnormal situations. HM wirelessly broadcasts emergency notifications to the surveillance center (SC) when an emergency situation is detected. ANGELAH followed the multi-attribute decision-making algorithm (MADM) to select a sufficient number of volunteers among individuals who wish to provide emergency assistance. The ANGELAH framework was designed and implemented for a case study, which involved an elderly subject suffering from severe vision impairment. The system aimed to track the subject's location to detect any abnormal behaviour such as falls or unusual activity/inactivity patterns. It also provided mobile groupware collaboration for assistance support. ANGELAH addressed the tracking of a single subject's location. Even though it aimed to expand the assistance providers, it did not consider the integration of available data sources and services in the smart home environment.

Information Technology for Assisted Living at Home [173], aka ITALH is a wireless home health system designed to remotely monitor patients and to alert caregivers in the event of accidental falls or acute illnesses. The project integrated a sensor architecture, called SensorNet, which is a heterogeneous wireless network that connects home sensors and sensors worn by subjects to a central home gateway and a mobile gateway. The sensors have processing capabilities that allow them to perform analysis, send alerts to the subject, and transmit occasional information to the central system when they detect a significant event. SensorNet supports Bluetooth and Zigbee wireless technologies, as well as hard-wired connectivity via USB. The project relies on home wireless (called fixed) sensors and wearable sensors. Fixed wireless sensors are connected to the wearable sensors and to the home and mobile gateway. The mobile gateway is in turn connected to the home gateway. Fixed sensors monitor the subject's environment and measure and analyze their motion. Uixed sensors were Telos Rev B Mote devices that support Zigbee (IEEE 802.15.4) and provide built-in temperature, humidity, and light sensing. For the wearable category, the system used the Berkeley fall sensor [184] which is an accelerometer-based fall sensing device. This sensor has a processor capable of analyzing incoming data and classifying motion events such as falls or other normal and abnormal events. Alert notifications related to abnormal situations are sent from the sensors to the home and mobile gateways, which fuse the data and determine an appropriate response. The two gateways connect the home network to the outside using the Internet and the telephone service to transmit alerts to caregivers.

In 173], the authors of ITALH focused more on using sensors worn by elderly subjects. The subject is equipped with 3 -axis accelerometers and a mobile phone camera to detect potential situations such as a fall. Acceleration data is processed locally and when a suspected fall is detected, the data is streamed to the camera phone using a Bluetooth connection. If there is no response from the subject to the initial phone request, the emergency center is then called and the fall detection data is streamed to them.

Another system, called COSAR (mobile context-aware activity recognition), was proposed in [151] using statistical and ontological reasoning on the Android platform. The system was developed to recognize ten ambulation activities like brushing teeth, strolling, and writing on a blackboard. Data is acquired by one accelerometer, embedded in a phone, and another one mounted on the subject's wrist. The subject's physical location was tracked using a GPS receiver. In their experiments based on this data, the authors selected the MLR (multiclass logistic regression) statistical learning algorithm as well as other techniques such as bayesian network, C4.5 decision tree, naive bayes, and SVM. MLR provided recognition rates higher than $80 \%$. However, the location dimension was the only context dimension that connected the proposed system to the context-awareness field.

The smart home care network (HS Care) 174] is a multi-modal sensing application for fall detection and subject location. The proposed system was designed to confirm fall situations and reduce the rate of false alarms. The system includes a user badge node equipped with accelerometers, voice transmission modules, cameras, and wireless communication functions such as radio-based triangulation and position estimation using network nodes. Basically, the system characterized fall detection using an accelerometer along with received signal strength (RSSI) between the subject's badge and network nodes in order to approximate their position. Image sensing and visionbased reasoning have been used to analyze situations and determine the subject's posture when an alert occurs. The badge node broadcasts an alarm message (SMS) to cameras when it detects changes in the accelerometers' data. The image processing algorithms run on local nodes in order to confirm the falls detection. If a fall is confirmed, the phone dials a stored number to make a call to the care center.

An RFID-Based indoor tracking system for the elderly (RFID-Track) was proposed in [27] to identify the person's location at home. The system comprises a wireless accelerometer which determines if the subject is walking, an active RFID reader with a signal strength function, and RFID reference tags placed in the environment to determine the probable locations of a subject.

The system defined two modules: the data manager and positioning manager. The data manager is used to filter out the noise from sensor data, establish the walking paths, and determine the number of walking steps using 
the three-axis acceleration values. The positioning manager accesses the environment data and uses the RSSI values as reference tags and walking paths to identify approximate regions. Then, based on the RFID values, number of steps and approximate regions, the positioning manager applies a genetic algorithm to compute the probable locations of the inhabitant. Similarly, an indoor U-Healthcare system proposed in [185] used RFID to estimate and track the elderly location. Sensed location data were associated with time slots and the length of time of stay in a given place. The system provides new useful information such as movement patterns, ranges, and frequencies.

RFID-based human behaviour modelling (RFID-Behavio was introduced in 90] to detect abnormal behaviour of elderly subjects according to their movements. The proposed system deployed several RFID active tags in the living environment, which included a notebook PC, a wireless AP and a mobile device (with a built-in RFID reader) carried by the elderly subject. Using deployed tags, the RFID reader detects RSSI values, which denote the distance between the tags and the reader. These values are recorded following the movement of the subject. A clustering technique was used to model subject movements within the range of normal behaviour. The system functionalities were divided into three parts: environment settings and data collection (i.e. transmitted and received RSSI values), data preprocessing (reducing RSSI noise and instability), and behaviour modelling by clustering. The latter considered two models for short-term behaviour and long-term behaviour. The short-term model is used to detect anomalies like falls in seconds, while the long-term model is used to detect long abnormalities like those related to bathroom activity and eating. Unfortunately, the system is not accurate enough to evaluate the subject's overall behaviour and performance regarding activities already carried out (e.g. the subject's bathroom and eating behaviour). Many devices were used to obtain only one signal, representing only one contextual dimension and, thus, cannot be used to assess the subject's overall human behaviour or to judge if the situation is really abnormal.

The fall detection system, called PerFallD [186], simply relies on mobile phones as a pervasive platform. This platform represents an indoor/outdoor environment system based on the hardware and software capabilities of mobile phones in terms of sensing (accelerometer sensors) and computing (executing the proposed detection algorithm).

Authors in 187] presented a method for movementbased activity recognition using the data recorded from sensors (infra-red sensors and/or magnetic contacts only) used to record movement sequences. The study targeted a limited set of activities and was based on the detection of sequences composed of a series of mobile and immobile states of the elderly subject. The main scenarios of mobile and immobile detection are achieved according to the values of two parameters: the time interval and the number of sensors. This detection method lacks the knowledge of real situations to determine whether the elderly subject properly carried out the activity. The Casattenta project presented in 23] is another ambient intelligence system which consists of fixed sensors and wearable commercial sensors. The system monitors movement recognition for elderly subjects in case of falls, immobility, or reaction incapacity. Wearable devices gather subject information and interact with fixed monitoring nodes based on the ZigBee wireless protocol.

A decision tree for an indoor localization algorithm, based on RSS measurements, was described in [188]. To reduce system complexity and location calculation time, the proposed distributed algorithm was based on simple Yes/No decisions. The platform consists of access points used with a Bluetooth low energy (BLE) chip on the local network and a wristband (mobile node) worn by the subject. All the BLE chips continuously broadcast location data, which enables the mobile node to approximate the actual location. By applying the decision tree on the four strongest RSS, gathered in one second, the mobile nodes can accurately locate themselves, thus solving network scalability and timing issues.

An unsupervised approach for human activity recognition (MHMMR), based on raw acceleration data of inertial wearable sensors was proposed in 87]. The proposition was motivated by the problem of automatic recognition of physical human activities using on-body wearable sensors, in a health-monitoring context. Human activities were classified using three accelerometer sensors attached to the chest, right thigh, and left ankle. Twelve ambulatory activities (dynamic, static and transitions). These are stairs down/up, standing, sitting down and related transitions, lying and related transitions, standing up, and walking. The proposed method is based upon the joint segmentation of multidimensional time series using a HMM in multiple regression contexts. Mainly, the advantage of this approach comes from the fact that the statistical model takes into account the regime (activity) changes over time through the hidden Markov chain. Moreover, using this statistical model, the learning is performed in an unsupervised framework using the expectation-maximization algorithm where no activity labels are needed. The proposed algorithm, however, assumes that the number of activities is known. On the other hand, the system attaches several sensors to the body, which overburdens the monitored subject, may decrease the subject's mobility and even obstruct the achievement of daily routines.

\subsection{Physiological Health Monitoring}

Physiological health applications are the most widely studied applications of ubiquitous healthcare systems in smart environments. These e-health applications first arose to address hospital related issues. However, with increasing related needs, the number of elderly subjects, and the development of information and communication technology, there has been an expansion of new systems into the home environment. Physiological applications emerged for 
the continuous remote monitoring of human physiological activities. They capture and transmit important vital sign parameters such as heart rate, blood pressure, arterial saturation of oxygen, body temperature, and blood glucose levels. These parameters are usually used for further evaluation and interaction in cases where abnormal conditions are detected. Hence, the system allows healthcare providers to monitor the medical condition of the subject and immediately provide appropriate services. In this category of applications, most studies and prototypes share the same functionalities and properties such as collect, capture, store and send context in terms of vital signs. Moreover, these applications face the same challenges collectively, such as reliability, intrusion, networking infrastructure, and energy consumption. Proposed systems are usually based on body sensor networks (BSN) which connect wearable sensing devices (such as shirts and belts) for data acquisition [40] 39]. In some cases, built-in sensors are designed for household objects like the so called $u$-Devices used in 189 for various objects (e.g. bed, couch and toilet). These devices measure the subject's vital signs and transmit their digitized health information ubiquitously.

An automatic in-home healthcare monitoring system, called EMUTEM, was proposed in [175]. The proposed telemonitoring system is a multimodal platform for the monitoring of elderly subjects and the detection of distress situations. The system consists of several sensors such as microphones, which are placed in all the rooms of the house. These allow for remote monitoring of the acoustical environment. The system also has a wearable device called RFpat, which measures physiological data (ECG) and also contact and temperature sensors, and a set of infrared sensors that detect the subject's presence, posture and movements. The system processes behavioural and physiological data, acoustical environmental data, environmental data, along with medical data. Sensors are connected to a home server. For audio monitoring, microphones are linked directly to the server through an external sound card. Infrared sensors are fixed to specific places in the house (walls and ceiling) and linked via radio frequency communication to a receiver connected to the server via a USB port. The wearable device is carried by the elderly subject and it collects physiological data continuously. It stores this data in an indoor reception base station via ZigBee. The novelty of this solution lies in the use of a multimodal data fusion approach based on fuzzy logic with a set of rules directed by medical recommendations. This multimodal fusion increases the reliability of the system and allows for the detection of several distress situations.

The authors of 39] and [41] developed a wearable context aware system (ETRI) and designed a tool for ubiquitous healthcare services. The work proposed an ontologybased context model, using the Web ontology language (OWL), and a reasoning engine to convert the sensor's preliminary context data into high-level contexts. The system consists of a set of wearable sensors, a watch and chest belt system including an electrocardiogram (ECG), a photoplethysmograph (PPG), a skin temperature sensor (SKT) and three axis acceleration sensors. A Zigbee communication module was used for measuring and transmitting pulsation and respiration. Bluetooth communications were integrated in order to link the system to commercial medical devices for measuring blood pressure, blood glucose levels, and body mass index. Wearable sensors and devices gathered biological signals and were connected to a personal digital assistant. The assistant held and processed biological signals and communicated with the home server for some additional processing such as database services. The proposed system is very similar to many modern applications existing in smartphones. Using various sensors, it allowed users to perform personal health checks based on their vital signs sent from sensors to the assistant. The results were then sent to the service provider. However, the system was still heavily dependent on human interaction for both subjects and service providers to determine the subject's medical health condition.

HeartToGo [121] is another mobile device-based wearable monitoring system. It was designed for continuous monitoring and real-time recording of ECG data. The system detects abnormal patterns related to the cardiovascular conditions and generates individualized health summary reports. HeartToGo proposed an artificial neural network-based machine learning technique to identify ECG features and to search for potential cardiovascular conditions. The system uses ECG sensors connected to a mobile device using a Bluetooth connection. Unfortunately, the system targeted single vital signs for the subject's physiological conditions independently of the subject's overall context.

A smart phone assisted chronic illness self-management system (SPA) was proposed in [133]. The proposed prototype aimed to provide continuous monitoring of elderly and disabled subjects, and to reduce, as much as possible, the involvement of healthcare professionals. Basically, the SPA system defined three main functions. First, it senses and monitors biomedical and environmental data. Second, it uses data analysis and data mining algorithms to identify time-series rules and relationships between collected data. Third, it automatically triggers on-line surveys and alarm notifications. The SPA architecture uses a smart phone with a GPS sensor to provide context-aware location data. It also uses a set of biosensors such as pulse oximeters and blood pressure meters, and a set of environmental sensors for sound, temperature, humidity, and light. Communication with sensors is achieved using Bluetooth. The smart phone works as a base station for the body area sensor network and communicates with a remote server either using a wireless LAN or a cellular network depending on availability. The remote server stores all the sensed data and deploys data mining algorithms to discover time-series patterns and rules. Algorithms are applied to identify unusual data such as dramatic changes in sensor readings. To validate collected data, SPA used 
questionnaire-based surveys sent via phone. Determined rules were mined after collecting sufficient data to assess the subject's stress, activity, and environment. However, the proposed system is still in the early stages and needs further proofing results.

A mobile healthcare monitoring system (Dongle), described in [176], was introduced to monitor a subject's physiological signs. Regarding the transmission of data, the network topology is based on a wireless LAN and the code division multiple access (CDMA) cellular network. The system's architecture consists of ECG sensors, which gather vital signs and directly transmit them to the server, in a medical center, via a 802.15.4-based access point. When the sensors are outside the LAN coverage and a mobile network coverage is available, the system stores and transmits ECG data to the cell phone using mounted wireless dongles. The cell phone is able to perform locally simple ECG analysis using the QRS detection algorithm [176]. When abnormal ECG signals are detected, related data is transferred to a server through the CDMA cellular network. However, the limitations of phone resources, processing, and memory prevent the performance of complex operations. In addition, the system considered only simple ECG analysis and provides basic information.

A user-friendly system was proposed in [177] to provide telemedicine services for the elderly and disabled at home. The system was designed to capture vital signs, some human activities and location, to allow caregivers interact with the subject and provide help. The system focused on health related data operations like data storage, update, and access. The architecture consists of physiological and ubiquitous sensors. The first category of sensors consists of wearable medical sensors including pulse oximeter and blood pressure sensors. These sensors were implanted on a wheel chair for capturing the vital signs of the elderly subject. The data is sent, upon request, to the caregiver. Sensor data is sent, through WiFi, to a database server. An emergency push button was designed for sending an emergency message through a GSM modem. The second category of sensors includes weight sensors, motion detectors, and light sensors. They are used to gather contextaware information to track the location and detect some activities. The system provides a Web interface used by the caregiver to monitor the elderly. However, the system is still limited regarding human interaction for both patients and caregivers. On the one hand, the subjects are obliged to use the wheel chair to enable vital sign collection. On the other hand, the caregivers still need to access the system, request vital signs data, and analyze medical reports. Concerning the activities of daily living, the project discussed modest context information by only focusing on scenarios where the elderly subject is going to the bathroom and kitchen at night only.

In [190], the proposed system defined a smart home ontology model (SHOM) used in a home monitoring framework. The objective was to provide autonomic support and assistance based on the elderly subject's context. The motivation for building a semantic model is to adopt a knowledge-based approach to capturing semantic data related to the elderly subject and their environment. The model was also built to perform as an autonomic decisionmaking system for the smart home. The ontology-based context model uses OWL and role-based reasoning using SWRL (semantic Web rule language) to understand the elderly subject's context. The architecture contains two commercial types of sensors: environmental wireless sensors (HyBus) and ECG monitoring medical sensor. The ECG sensor is composed of a body sensor part and a receiving base. The receiving base is connected to an environmental wireless sensor using a USB port. The sensor part senses the heartbeat and sends an ECG signal to the base using RF. Overall, the system focused more on the analysis of collected data (for autonomic decisions) than on the networking techniques. The proposed SHOM model requires further improvements in order to provide smart decisions strongly related to the subject's behaviour.

The AlarmNet prototype, presented in [22], is a wireless medical sensor network system designed to monitor the physiological condition of the subject, along with some environmental conditions. Five main components were used: mobile body networks, embedded sensors, gateway (AlarmGate), back-end, and user interfaces. The mobile body network is composed of sensors used to provide physiological monitoring. These sensors, like ECG, pulse, blood pressure and oxygen saturation, were developed in the CodeBlue project [191]. Embedded sensors are used to provide environmental information and subject location tracking using temperature, dust, light, motion and tripwire sensors. The AlarmGate connects various wireless sensors to the IP network. Data is streamed, either directly or in multi-hops, to the AlarmGate gateways for storage, analysis, or distribution to user interfaces. The major aspects of the AlarmNet system are privacy, energy management, data access, and security. Data is stored long-term in the back-end, to analyze and learn behaviour patterns. To do this, the system uses the Circadian activity rhythms (CAR) algorithm 22]. The graphical user interface was used to allow caregivers to query sensor data and display physiological and environmental measurements.

The work presented in [192] focused on the remote monitoring of a particular kind of population which involves aged or unwell subjects, who move back to their homes from hospital or care facilities, yet still need health monitoring for some medical condition and/or fall detection. It is a personalized smarthome care system, which uses smartphones, IP cameras, lights sensor and some commercial devices (3-Axis accelerometers, weight sensors and blood pressure sensors). Diverse network technologies such as wired, Bluetooth, Wi-Fi, and X10 communications were integrated together. Using these technologies, sensors and devices send the subject's vital signs (blood pressure and weight) and fall detection data to caregivers. Unfortunately, subjects still have to perform many tasks and interact with the system. For instance, they have to weigh 
themselves, measure their blood pressure every morning and charge the smartphone battery every night. The result of such permanent constraints is a system, which is ineffective regarding context-aware healthcare monitoring.

The healthcare monitoring system, U-health [189], detects physical and movement activities using unconstrained measurement devices. Devices are built-in and IR sensor type devices. The built-in sensors (bed, sofa, and toilet seat) detect small changes to the subject's physical activity such as heart and respiration rates and blood pressure via sensitive load cell sensors (ECG, and PPG). IR motion sensors detect the subject's movements. Raw data, collected from these devices, are sent to a home server using a Bluetooth wireless network. The system does not define a method for analyzing data stored in the system longterm. A home telemonitoring system that aims to integrate the monitoring of vital signs was introduced in [193]. The system supports several Bluetooth profiles and protocols which helps the integration of heterogeneous medical devices. It stores physiological parameters using the personal healthcare monitoring report (PHMR) available for healthcare professionals. The core of the system is based on an Android application hosted on a minicomputer connected to the home TV. Vital signs like blood pressure, pulse rate, body weight and blood glucose are collected using a medical device installed in the home. The medical device sends the data to the application used by the general practitioners, who send feedback to the subjects through a web interface.

\section{Ongoing Challenges and Open Issues}

Providing healthcare and monitoring services in smart spaces is a huge domain, spanning several concepts and raising multiple challenges and issues. The essential goal of these systems is to collect highly relevant contextual data, to provide evaluations, diagnosis, treatments, quick decisions, and relevant services. A lot of issues and challenges still exist. but improvements in software applications, hardware, and network communications need to happen before they can be resolved. Based on previous surveys, we discuss in this section the most important issues and challenges facing health monitoring systems.

\subsection{Monitoring Accuracy}

Monitoring and evaluating human subjects, in particular the elderly, is a complex task due to the complexity of performing diverse daily activities. behaviour is subjective and changes from one subject to another. it can even change for the same person depending on mood and health conditions. Most of the current studies related to monitoring of activities focus on relatively simple activities that are performed over short periods within a laboratory environment. In the real world, however, complex activities and those executed over a long-term period are clearly more difficult to manage in terms of collection and analysis of relevant data. Moreover, activities which have several sub-actions can be performed in different ways and diverse order.

Long-term monitoring is useful for detecting the real behavioural patterns of a person in a smart home. In existing research experiments, data for the activities of a single user is collected and analysed, in the smart environment. However, in reality, daily activities can be concurrently performed by multiple subjects who share the same space.

Existing interactions between subjects can hide the real ability of the person to perform ADL and increase the complexity of monitoring. Therefore, considering the monitoring of multiple subjects is a more challenging and difficult task. In addition, most research in this realm is focused on recognizing activities rather than evaluating whether the subject performs these activities correctly or not. According to these challenges, the accuracy and robustness of the results are still relatively far from the real world, in which human behaviour is mysterious and variable.

We believe that there is a need for more effort to improve the design and development of monitoring systems for elderly subjects in smart environments. In such systems and solutions, appropriate sensors should be carefully selected to capture rich and relevant data for single or multiple subjects. The system should identify suitable methods and techniques that can effectively understand complex and ever-changing human behaviour. Such systems must be tested over long periods to ensure their effectiveness in terms of analysis, accuracy, and adaptability of the proposed monitoring. Hence, the system can provide appropriate services based on the subject's context.

\subsection{Context-Awareness}

A healthcare context-awareness system provides users with relevant, suitable, and personalized applications, interfaces and services matching their context and its evolution. A full overview of a subject's context allows for the system to dynamically determine the subject's real needs in terms of assistance. In HMS, it is important to correctly interpret data and events to extract a coherent high-level abstraction of contextual data. In addition to classic monitoring techniques, emergency situations and deterioration in health conditions should be reliably detected as early as possible. Overall, the design of context-aware healthcare applications faces two major challenges, which are data acquisition (which information is worth capturing) and data analysis techniques including the presentation of contextual information and services [194]. The representation and context-aware interpretation of data is the key aspect influencing the success of a health monitoring system. An accurate detection of emergency situations allows for rapid interventions and, therefore, improves the quality of healthcare for subjects. For instance, according to the subject's context and condition, not all of their detected falls represent critical cases or need a reaction by the system or caregiver. Regardless of the fact that many existing systems fail to reach a high degree of context-awareness, 
full and complete contextual information collection is still a critical open issue in need of further attention.

The design of context-aware monitoring systems should consider observation, interpretation, and reasonning about the patient's conditions from various perspectives: behavioural, physiological, and environmental. The system must consider all the relevant contextual dimensions, which consist mainly of location, time, objects, posture, frequency and performed human activities. Historical data including health records (like diagnoses, diseases, and treatments), current daily behaviour and previous changes, and the environment conditions (like temperature and humidity) also influence the system's intelligence. Healthcare systems must be able to determine what, when and how to monitor, gather and analyze data related to the subject's context [195]. On another front, the same context data should be available on several distribution components using different network technologies. This enables different caregivers to obtain necessary contextual information from the infrastructure using the available network that can often vary in a pervasive environment. A context-aware handover mechanism was proposed in [70] to provide the capability of selecting the suitable network interface for data transfer.

Although a variety of sensor technologies are widely available today, context-aware systems for health services do not fulfil the desired requirements. There are many aspects, which need further work. In spite of several studies and healthcare applications, which have been proposed in context-aware computing, there are still open issues and challenges such as the lack of recommendations for functional needs of the context, the gap between fundamental context representation and actual context awareness prototypes and the difficulties in building efficient systems to simulate the human perspective 21].

\subsection{Human factors}

One important issue is the consideration of human factors such as human acceptability of a given system and the degree to which human interactions are supported by the proposed system. The consideration of human factors and ergonomics (HFE) and human-computer interaction (HCI) helps to optimize human well-being, safety, and overall system performance [196]. From a design level, these factors help us understand the interactions between the subject and the system components. This relates to caregivers (occupational ergonomics) and care receivers (subjects).

In the healthcare realm, the need to characterize these interactions have been addressed by studies, such as [197] and [198], in order to fulfill the needs of the elderly. Human factors should be considered when designing systems that improve the quality of healthcare. Consider the minimization of errors, which improves the system's adoption rate by the subject. Specifically, consider factors that aim to achieve effectiveness, efficiency, and subject satisfaction in the design of a health monitoring system 198]. This consideration is of paramount importance, especially for an aging population that does not necessarily have any desire to use modern and complex technologies. Added to this, the elderly suffer from age-related health issues (e.g. decline of cognitive abilities), which prevent them from adopting and learning new technological solutions. These kinds of factors have been considered in the context of designing monitoring solutions for the elderly in studies such as in [42] 198] and 199].

For caregivers, further development of HMS involves the provision of ambulatory and less residential care services carried out by isolated workers or small teams, which may complicate the working system. HMS should be well understood and integrated as an easy tool that minimizes the caregiver's burden and facilitates interactions between stakeholders. Moreover, the design of HMS should improve the quality of human well being and avoid some negative work-related effects such as those related to patienthandling activities, turnover or compassion fatigue, stress, violence, and the impact of aging on caregivers themselves 197 198.

HMSs promote human safety in healthcare for the various stakeholders including the elderly, caregivers, emergency personnel, etc. This factor is of paramount importance in healthcare and remains a concern. It has been studied in a number of surveys such as [197], 198], and [200]. The authors in 200] provide five major recommendations when considering safety factors: build capacity among caregivers to understand the HFE, integrate HFE into medical technologies, increase the number of human factors and ergonomic practitioners in healthcare institutions, expand investments in improvement efforts informed by HFE, and support interdisciplinary research. For subjects, these factors are perceived as the most useful of HMS systems and are usually the main causes of subjects leaving their homes and moving to care facilities. HMSs are expected to continually provide care services, like emergency help and accident detection, especially when caregivers are away. HMSs should ensure the safety of subjects and caregivers in various settings and prevent violence and abuse from any source including from subjects with mental disorders. HMS could may rely on existing tools, ranging from simple presence sensors to video surveillance systems with advanced functionalities such as movement pattern tracking and recognition, real-time alerts, etc. Biometric sensors can also be used for detecting the signs of stress and aggression especially in healthcare institutions.

We believe that usability, acceptability, and safety are the most important factors to be considered for both monitored subjects and caregivers in HMS [42] [197, 198, 200202. Unfortunately, these factors have been ignored by the majority of works we have examined to date and are, thus, worthy of future attention.

\subsection{Heterogeneity}

Health applications need a variety of contextual data which are gathered using different and heterogeneous sources. In HMS, the heterogeneity is mainly observed in two forms: 
heterogeneity of data and heterogeneity of sensors. Data is collected from multimodal sensors, which are heterogeneous in format, structure and semantics. Thus, datasets are difficult to share and reuse due to the lack of formal descriptions. Although the efforts made to develop sensor data models, used to describe semantics, there is a need to normalize the sensor data modelling to represent heterogeneous data sources. The integration of several heterogeneous sensors that operate at different frequencies and use different network protocols raises the problem of interoperability. There are numerous studies, which explored related heterogeneity issues over HMS 203 204]. In [204], the main issues identified were the integration of heterogeneous devices in a single network, the lack of a common architecture supporting different devices and the difficulty of integrating HMS with other systems.

Prior attempts to solve the heterogeneity problem have been made and various solutions were explored. One of the proposed solutions at the application level is middleware design. As explained previously, middleware helps to manage heterogeneity and provide abstraction between applications and sensors to simplify the application development. For instance, the MPIGate gateway [203] integrates multi-platform systems in a unified framework and offers modular and transparent data access. The unified framework allows the interconnection of heterogeneous networks and existing middleware solutions. Another middleware, proposed for wireless medical body area networks [205], was able to provide a multiplexing of biometric readings using specialized sensors distributed in different parts of the body.

The combination of sensors and devices in HMS can generate a high level of interference. The interferences and overlap of ranges caused by the coexistence of several wireless networks can significantly affect the reliability of the healthcare network system and hence the availability of critical data [206] [207]. Existing experiments show the impact of interference on received data rate [208] and increased delays and power consumption [209]. Some existing strategies were proposed to address these related issues, such as the coexistence of ZigBee and Wifi for HMS [210], the coexistence between Bluetooth LE and technologies using the same spectrum [211], the overlapping of wireless channels using allocation schemes and algorithms [212]. The schemes proposed in [213] aim to provide a reliability in the transmission of critical health monitoring data.

Nevertheless, experiments in previous studies have demonstrated other problems such as those related to the delays and consumption of resources. There is still a need to find new schemes and strategies that generally guarantee the support of heterogeneity with good performance, the simplification of architectures, protocols and normalization of data formats and presentations.

\subsection{Availability and Reliability}

Making appropriate health data accessible in a timely manner is another critical issue for HMS. The availability of health information can be influenced by the reliability of data delivery, which in turn could negatively impact a subject in a critical situation. Data reliability on wireless networks in health infrastructures is subject to a number of factors. Such factors are network coverage, device range, power availability, routing protocols, and network or devices failures. Reliability issues have been already classified into three categories: data measurement, data communication, and data analysis [54]. Numerous studies have addressed issues in wireless health monitoring, for instance, using redundancy elimination [214] and data cleaning [54].

A data coding and transmission method was proposed in [214]. It includes two coding stages: compression and inflation. The first stage is used to compress naturally redundant information and the second is used to inject artificial redundancy into wireless transmission to enhance transmission reliability. Hence, after data sensing and coding, wireless sensor nodes transmit the coded data instead of raw sensor data. The authors in [54] described specific healthcare monitoring issues related to software including data collection, data fusion, and data analysis. They proposed, as aa result, an architecture for handling data cleaning, data fusion, and context and knowledge generation for more reliable data analysis.

Compared to traditional infrastructures, the number and complexity of sensors and employed methods in HMS is significantly higher. In addition, the heterogeneity of data sources, as previously discussed, adds a greater level of complexity that must be considered to ensure data, regardless of its source, is reliable. Existing sensors are still limited in terms of hardware and software capabilities and, thus, are subject to failure at any time. Consequently, considering robust fault-tolerant strategies is essential for HMS.

\subsection{Data Transmission}

A common challenge in the development of monitoring systems, especially in WBAN applications, is the selection method for transferring sensor data to back-end servers for processing and analysis.

In networking, data transmissions are categorised into four schemes: multicast, broadcast, unicast, and anycast. Existing HMS often use multicast or broadcast schemes to increase reliability and optimize data transmission. Both schemes simultaneously deliver packets to multiple receivers. Hence, frequent transmissions lead to a high level of network traffic and transmission delay. The unicast scheme, where packets are delivered to a single receiver, has the least traffic overhead but requires additional procedures to find an alternate receiver in case of transmission failure. The anycast communication mode is a new routing approach where data packets are routed to the nearest receiver. This scheme has lower traffic overhead compared 
to broadcast and multicast and it is more reliable than unicast for finding new receivers. However, anycast increases the complexity of network routing and the devices employed by the scheme.

Improving message delivery over wireless ad hoc networks in HMS by using several routing schemes was discussed in [215]. In 216], a reliable transmission protocol, based on the anycast routing scheme, was proposed for wireless monitoring. Sensor nodes select the nearest data sink in a ZigBee network, to transmit messages with vital sign data. The proposed protocol uses a ZigBee device (accelerometer) for ECG and fall monitoring and indoor positioning. When the accelerometer detects a fall, the subject's position and ECG signals are transmitted to a data sink. In the case of a network path failure, the transmission path is rebuilt from the last node before the failure link. Hence, it provides reliable communication and reduces transmission latency as well as reducing traffic overhead. The wireless patient monitoring system framework, discussed in [217], involves the processing of vital signs, routing schemes, and protocols for message delivery. The performance results show that reliable message delivery and low monitoring delays can be achieved by using multicast or broadcast routing schemes.

There are broader considerations arising from this challenge including transmission technologies, determining the amount of transmitted data, regular packet size, transmission frequencies. All of these considerations have a major impact on the system's effectiveness, availability, reliability, network traffic and energy consumption. In HMS, the reliability of data transmissions depends on the range of devices used by the subject themselves, cooperating devices integrated into the HMS architecture, routers, and target receivers.

\subsection{Security and Privacy}

In HMS, security requirements are the same as those of traditional networks, such as availability, confidentiality, integrity, access control, and authentication [56] [218]. Healthcare monitoring systems rely heavily on several technologies that can pose security threats or be used in attacks such as eavesdropping and modifying medical data, location and activity tracking, forging alarms on medical data, denial of service, physical tampering of devices, and jamming attacks 219]. Moreover, deploying wireless technologies in healthcare systems without considering security requirements often makes subjects vulnerable to privacy issues [220]. Privacy should also be a major concern in healthcare applications. The transmission of personal data over the Internet and wireless media could pose serious threats to the privacy of a subject.

Security in health monitoring systems is a critical issue, which involves many components and processes: sensors and devices, data collection, and communication. In HMS, applying security strategies should take into consideration the constraints of the HMS environment, such as the battery life of sensors, the storage of sensor data and historic records, and the network technologies employed. Since HMS deal with sensitive and critical subject data, existing cryptographic methods (like encryption and authentication) can be used to provide subject privacy and security against attacks. Robust cryptography techniques need, however, wide computation and resources. Therefore, identifying the appropriate encryption strategy remains challenging. HMS usually involve huge data collection and processing for continuous monitoring and sensor control. Therefore, there is a need to define optimal methods that can provide maximum security and privacy with minimal resource usage. In [221], the authors have raised several interesting questions regarding privacy and security concerns. For instance, where health data should be stored, who can view a subject's medical record, to whom should this information be disclosed without the subject's consent, and who will be responsible for maintaining the data.

\subsection{Intrusiveness}

Improving quality of life is one of the challenges of HMS. Some proposed applications, like BSN, require subjects to constantly wear and carry sensors. This can become a cumbersome task for subjects. More efforts needs to be made to ensure no violations to the lives of subjects occur. Some studies have addressed part of this problem with mobility and portability solutions. For instance, TV sets were used to provide healthcare services in [42], a smart shirt which continuously measures ECG and acceleration signals was introduced in [40], and a watch for motion capture in body area sensor networks was introduced in [38]. Unfortunately, the scope of the provided health services in such attempts and existing propositions is still limited and further efforts must be made.

\subsection{Power Consumption}

Energy consumption is another important issue of sensor network applications especially in BSN. The life cycle of HMS can be negatively affected by the limited battery life of sensors. Battery replacement and charging in most existing BAN systems is inefficient and cumbersome for monitored subjects especially in architectures with several sensors. Normally, the battery life of any communication device depends on its duty cycle, transmission range, and the way in which communication channels are used. In order to save energy with the widely used MAC protocol, sensor nodes can periodically turn On/Off their radio interface with a centrally assigned time slot [222]. However, the traditional use of MAC protocols has shown inadequate network throughput and delay performance at varying traffic [5]. Recently, in medical sensor networking, there has been a tendency to use the new low-power standard, Bluetooth low energy (BLE). BLE allows sensors and devices to operate for over a year without recharging or changing battery [223] [51]. Despite the the low-power issue being addressed in many previous studies, it is still a significant issue that needs further research in HMS. 


\section{Conclusion}

The research fields of health monitoring and smart spaces are extremely active. A wide range of approaches for monitoring and evaluating subjects are currently being investigated. Health monitoring systems have emerged as a promising solution to the problem of an aging population, by providing context-aware e-health services. In this study, we provide a comprehensive survey of context-aware computing in healthcare systems for subjects, in particular for the elderly. Our objective is to highlight the requirements, technologies, and challenges in developing HMS in a smart environment. We provide a set of guidelines that can provide an understanding of the issues that need to be addressed to improve context-awareness in healthcare services. The essential components required for HMS have been described in detail. Moreover, we present a consolidated picture of the essential functions offered by HMS for monitoring and detecting the subject's behaviour including concepts, approaches and processing methods. We provided a survey of recent research, evaluated the state of the art of monitoring systems and healthcare applications, and discussed the research challenges for future healthcare applications.

Although in recent years, there has seen a plethora of impressive works on health monitoring, we identified the weaknesses of existing systems, there main challenges and a number of open issues. To our knowledge, no existing approach considers, in a systematic way, the subject's context for designing context-aware e-health ecosystems. In the area of sensing and health monitoring, a lot of existing work take whatever is measurable and consider it as the subject's context. They then use modelling techniques to detect anomalies and when abnormal behaviour is detected, the subject's health status is deemed to have changed. Health and behavioural systems for the elderly have been defined based on the availability of sensing rather than the use of medical/expert/geriatric knowledge and a good understanding of the subject's context. The lack of such considerations leads to high uncertainty in the success and adoption of such projects. For efficient health monitoring, the achievement of basic activities of daily living (ADL) and the analysis of health conditions must be considered, otherwise, any effort in improving the quality of life and healthcare for persons using smart home technologies would be ad-hoc and cannot be expected to provide desired results.

Context-aware assisted living systems must have full visibility of the subject's context. This visibility includes the ADL performances and anomalies detection [84]. The important challenge in such intelligent environments is to determine: what, when and how to monitor, gather and analyze data related to the person's context [195]. Most of the health monitoring approaches and context-aware assisted living systems tend to apply unconditional processing of all collected data. This continuous monitoring approach is resource intensive regardless of the subject's con- text. The adoption of this approach raises several issues such as network collapse, data transmission failure, energy consumption, important computational costs, and loss of priorities in processing and making decisions that should be relevant and quick. The same situation occurs with physical monitoring applications, which assume a uniform time interval of data sensing and analysis.

In order to enhance the reliability of health data transmission and the availability of highly relevant contextual information, it is necessary to define efficient data aggregation algorithms and relevant data filtering mechanisms for application in conditional monitoring schemes. Furthermore, several methods can be applied for detecting human behaviour and for extracting context in smart health environments. These approaches typically require a substantial amounts of training data, in order to learn and detect human behaviour and context. Therefore, such methods can provide good performance but at a high computational cost. As a result, there is still a need to improve intelligent processing, which combines an optimal contextaware monitoring cost with accurate behaviour detection, in health monitoring systems. All the while, bearing in mind the importance of considering human factors for the overall success of these systems.

\section{References}

[1] United Nations Population Fund (UNFPA), Ageing in the Twenty-First Century: A Celebration and A Challenge, http://www.unfpa.org/publications/ ageing-twenty-first-century [Accessed: May 2016] (2012).

[2] Global Health Workforce Alliance and World Health Organization, A Universal Truth: No Health Without a Workforce, http://www.who.int/workforcealliance/knowledge/ resources/hrhreport2013/en/ [Accessed: May 2016] (2013).

[3] M. Alam, M. Reaz, M. Ali, A review of smart homes-past, present, and future, IEEE Transactions on Systems, Man, and Cybernetics, Part C: Applications and Reviews 42 (6) (2012) 1190-1203.

[4] M. Baig, H. Gholamhosseini, Smart health monitoring systems: an overview of design and modeling, Journal of medical systems 37 (2) (2013) 1-14.

[5] M. Chen, S. Gonzalez, A. Vasilakos, H. Cao, V. Leung, Body area networks: A survey, Journal of Mobile Networks and Applications 16 (2) (2011) 171-193.

[6] K. Peetoom, M. Lexis, M. Joore, C. Dirksen, L. Witte, Literature review on monitoring technologies and their outcomes in independently living elderly people, Disability and Rehabilitation: Assistive Technology 10 (4) (2015) 271-294.

[7] P. Rashidi, A. Mihailidis, A survey on ambient-assisted living tools for older adults, IEEE Journal of Biomedical and Health Informatics 17 (3) (2013) 579-590.

[8] B. Reeder, E. Meyer, A. Lazar, S. Chaudhuri, H. Thompson, G. Demiris, Framing the evidence for health smart homes and home-based consumer health technologies as a public health intervention for independent aging: A systematic review, International journal of medical informatics 82 (7) (2013) 565579 .

[9] A. Dey, Understanding and using context, Personal and ubiquitous computing 5 (1) (2001) 4-7.

[10] L. Sanchez, J. Lanza, R. Olsen, M. Bauer, M. Girod-Genet, A generic context management framework for personal networking environments, in: IEEE International Conference on Mobile and Ubiquitous Systems-Workshops, 2006, pp. 1-8. 
[11] M. Heeren, et al., Active at night, sleepy all day-sleep disturbances in patients with hepatitis c virus infection, Journal of hepatology 60 (4) (2014) 732-740.

[12] M. Pantic, A. Pentland, A. Nijholt, T. Huang, Human computing and machine understanding of human behavior: a survey, in: Artifical Intelligence for Human Computing, Springer, 2007, pp. $47-71$.

[13] N. Ryan, J. Pascoe, D. Morse, Enhanced reality fieldwork: the context-aware archaeological assistant, in: Computer Applications and Quantitative Methods in Archaeology, 1997, pp. 269-274.

[14] B. Schilit, N. Adams, R. Want, Context-aware computing applications, in: IEEE Workshop on Mobile Computing Systems and Applications, IEEE, 1994, pp. 85-90.

[15] G. Abowd, A. Dey, P. Brown, N. Davies, M. Smith, P. Steggles, Towards a better understanding of context and contextawareness, in: Handheld and ubiquitous computing, Springer, 1999, pp. 304-307.

[16] G. Abowd, E. Mynatt, Charting past, present, and future research in ubiquitous computing, ACM Transactions on Computer-Human Interaction 7 (1) (2000) 29-58.

[17] P. Brown, Triggering information by context, Personal Technologies 2 (1) (1998) 18-27.

[18] N. Ryan, Mobile computing in a fieldwork environment: Metadata elements, project working document (1997).

[19] M. D. Rodríguez, J. Favela, An Agent Middleware for Ubiquitous Computing in Healthcare, Springer, 2008, Ch. 6, pp. $117-149$.

[20] C. Perera, A. Zaslavsky, P. Christen, D. Georgakopoulos, Context aware computing for the internet of things: A survey, IEEE Communications Surveys and Tutorials 16 (1) (2014) 414-454.

[21] N. Bricon-Souf, C. Newman, Context awareness in health care: A review, International journal of medical informatics 76 (1) (2007) $2-12$.

[22] A. Wood, et al., Context-aware wireless sensor networks for assisted living and residential monitoring, IEEE Journal on Network 22 (4) (2008) 26-33.

[23] E. Farella, M. Falavigna, B. Riccò, Aware and smart environments: The casattenta project, IEEE Transactions on Biomedical Engineering 41 (11) (2010) 697-702.

[24] C. Poon, B. Lo, M. Yuce, A. Alomainy, Y. Hao, Body sensor networks: In the era of big data and beyond, IEEE Reviews in Biomedical Engineering 8 (2015) 4-16.

[25] C. Franco, J. Demongeot, C. Villemazet, N. Vuillerme, Behavioral telemonitoring of the elderly at home: Detection of nycthemeral rhythms drifts from location data, in: International Conference on Advanced Information Networking and Applications Workshops, 2010, pp. 759-766.

[26] N. Noury, T. Hadidi, Computer simulation of the activity of the elderly person living independently in a health smart home, Computer Methods and Programs in Biomedicine 108 (3) (2012) 1216-1228

[27] C. Hsu, J. Chen, A novel sensor-assisted rfid-based indoor tracking system for the elderly living alone, Journal of Sensors 11 (11) (2011) 10094-10113.

[28] W. Jih, J. Hsu, C. Wu, C. Liao, S. Cheng, A multi-agent service framework for context-aware elder care, in: Workshop on Service-Oriented Computing and Agent-Based Engineering, 2006, pp. 61-75.

[29] T. Hori, Y. Nishida, Ultrasonic sensors for the elderly and caregivers in a nursing home, in: International Conference on Enterprise Information Systems, 2005, pp. 110-115.

[30] N. Suryadevara, S. Mukhopadhyay, R. Wang, R. Rayudu, Forecasting the behavior of an elderly using wireless sensors data in a smart home, Engineering Applications of Artificial Intelligence 26 (10) (2013) 2641-2652.

[31] L. Gao, A. Bourke, J. Nelson, Evaluation of accelerometer based multi-sensor versus single-sensor activity recognition systems, Medical Engineering and Physics 36 (6) (2014) 779785 .
[32] M. Jiang, H. Shang, Z. Wang, H. Li, Y. Wang, A method to deal with installation errors of wearable accelerometers for human activity recognition, Physiological measurement 32 (3) (2011) 347-358.

[33] A. Mannini, S. Intille, M. Rosenberger, A. Sabatini, W. Haskell, Activity recognition using a single accelerometer placed at the wrist or ankle, Medicine and science in sports and exercise 45 (11) (2013) 2193-2203.

[34] J. Varkey, D. Pompili, T. Walls, Human motion recognition using a wireless sensor-based wearable system, Personal and Ubiquitous Computing 16 (7) (2012) 897-910.

[35] L. Kau, C. Chen, A smart phone-based pocket fall accident detection system, in: International Symposium on Bioelectronics and Bioinformatics, 2014, pp. 1-4.

[36] D. Ashbrook, T. Starner, Using gps to learn significant locations and predict movement across multiple users, Personal and Ubiquitous Computing 7 (5) (2003) 275-286.

[37] L. Liao, D. Patterson, D. Fox, H. Kautz, Learning and inferring transportation routines, Artificial Intelligence 171 (5-6) (2007) 311-331.

[38] A. Barth, M. Hanson, H. Powell, J. Lach, Tempo 3.1: A body area sensor network platform for continuous movement assessment, in: International Workshop on Wearable and Implantable Body Sensor Networks, 2009, pp. 71-76.

[39] D. Kang, H. Lee, E. Ko, K. Kang, J. Lee, A wearable context aware system for ubiquitous healthcare, in: IEEE International Conference on Engineering in Medicine and Biology Society, 2006, pp. 5192-5195.

[40] Y. Lee, W. Chung, Wireless sensor network based wearable smart shirt for ubiquitous health and activity monitoring, Journal of Sensors and Actuators B: Chemical 140 (2) (2009) 390-395.

[41] D. Kang, K. Kang, H. Lee, E. Ko, J. Lee, A systematic design tool of context aware system for ubiquitous healthcare service in a smart home, in: International Conference on Future Generation Communication and Networking, 2007, pp. 49-54.

[42] T. Lemlouma, M. A. Chalouf, Smart media services through tv sets for elderly and dependent persons, Springer Lecture Notes of the Institute for Computer Sciences, Social Informatics and Telecommunications Engineering 61 (2013) 30-40.

[43] M. V. et al., The sweet-home project: Audio technology in smart homes to improve well-being and reliance, in: International Conference of the IEEE Engineering in Medicine and Biology Society, 2011, pp. 5291-5294.

[44] D. Brulin, Y. Benezeth, E. Courtial, Posture recognition based on fuzzy logic for home monitoring of the elderly, IEEE Transactions on Information Technology in Biomedicine 16 (5) (2012) 974-982.

[45] B. Schiele, M. Andriluka, N. Majer, S. Roth, C. Wojek, Visual people detection: Different models, comparison and discussion, in: IEEE International Conference on Robotics and Automation, 2009, pp. 1-8.

[46] T. Taleb, D. Bottazzi, M. Guizani, H. Nait-Charif, Angelah: a framework for assisting elders at home, IEEE Journal on Selected Areas in Communications 27 (4) (2009) 480-494.

[47] G. Sacco, et al., Detection of activities of daily living impairment in alzheimer's disease and mild cognitive impairment using information and communication technology, Clinical interventions in aging 2012 (7) (2012) 539-549.

[48] R. Robles, T. Kim, A review on security in smart home development, International Journal of Advanced Science and Technology 15 (2010) 13-22.

[49] Z. Wang, X. Xu, Smart home $\mathrm{m} 2 \mathrm{~m}$ networks architecture, in: International Conference on Mobile Ad-hoc and Sensor Networks, 2013, pp. 294-299.

[50] A. Omre, Bluetooth low energy: Wireless connectivity for medical monitoring, Journal of Diabetes Science and Technology 4 (2) (2010) 457-463.

[51] Y. Park, H. Cho, Transmission of ecg data with the patch-type ecg sensor system using bluetooth low energy, in: International Conference on ICT Convergence, 2013, pp. 289-294. 
[52] J. Pascoe, Adding generic contextual capabilities to wearable computers, in: International Symposium on Wearable Computers, Digest of Papers, IEEE, 1998, pp. 92-99.

[53] S. Lee, J. Chang, S. Lee, Survey and trend analysis of contextaware systems, Information-An International Interdisciplinary Journal 14 (2) (2011) 527-548.

[54] H. Lee, K. Park, B. Lee, J. Choi, R. Elmasri, Issues in data fusion for healthcare monitoring, in: International Conference on PErvasive Technologies Related to Assistive Environments, 2008, p. 3.

[55] D. Halperin, T. Kohno, T. Heydt-Benjamin, K. Fu, W. Maisel, Security and privacy for implantable medical devices, IEEE Pervasive Computing 7 (1) (2008) 30-39.

[56] M. A. Ameen, J. Liu, K. Kwak, Security and privacy issues in wireless sensor networks for healthcare applications, Journal of medical systems 36 (1) (2012) 93-101.

[57] A. Mileo, D. Merico, R. Bisiani, Support for context-aware monitoring in home healthcare, Journal of Ambient Intelligence and Smart Environments 2 (1) (2010) 49-66.

[58] G. Hackmann, W. Guo, G. Yan, Z. Sun, C. Lu, S. Dyke, Cyberphysical codesign of distributed structural health monitoring with wireless sensor networks, IEEE Transactions on Parallel and Distributed Systems 25 (1) (2014) 63-72.

[59] P. Bagga, R. Hans, Applications of mobile agents in healthcare domain: A literature survey, International Journal of Grid and Distributed Computing 8 (5) (2015) 55-72.

[60] W. Omar, A. Taleb-Bendiab, E-health support services based on service-oriented architecture, IT Professional 8 (2) (2006) 35-41.

[61] R. Rodrigues, et al., Monitoring intelligent system for the intensive care unit using rfid and multi-agent systems, in: IEEE International Conference on Industrial Engineering and Engineering Management, 2012, pp. 851-855.

[62] S. Rodriguez, C. Zato, J. Corchado, T. Li, Fusion system based on multi-agent systems to merge data from wsn, in: International Conference on Information Fusion, 2014, pp. 1-8.

[63] M. Papazoglou, Service-oriented computing: concepts, characteristics and directions, in: Proceedings of the Fourth International Conference on Web Information Systems Engineering, 2003, pp. 3-12.

[64] Y. Trinugroho, F. Reichert, R. Fensli, A soa-based ehealth service platform in smart home environment, in: IEEE International Conference on e-Health Networking Applications and Services, 2011, pp. 201-204.

[65] A. Forkan, I. Khalil, Z. Tari, Cocamaal: A cloud-oriented context-aware middleware in ambient assisted living, Future Generation Computer Systems 35 (2014) 114-127.

[66] V. Vaidehi, R. Bhargavi, K. Ganapathy, C. S. Hemalatha, Multi-sensor based in-home health monitoring using complex event processing, in: International Conference on Recent Trends In Information Technology, 2012, pp. 570-575.

[67] A. Benharref, M. Serhani, Novel cloud and soa-based framework for e-health monitoring using wireless biosensors, IEEE Journal of Biomedical and Health Informatics 18 (1) (2014) 46-55.

[68] P. Garcia-Sancheza, J. Gonzaleza, A. P. A. Moraa, Deploying intelligent e-health services in a mobile gateway, Expert Systems with Applications 40 (4) (2013) 1231-1239.

[69] A. Triantafyllidis, V. Koutkias, I. Chouvarda, N. Maglaveras, A pervasive health system integrating patient monitoring, status logging, and social sharing, IEEE Journal of Biomedical and Health Informatics 17 (1) (2013) 30-37.

[70] A. Soomro, R. Schmitt, A framework for mobile healthcare applications over heterogeneous networks, in: 13th IEEE International Conference on e-Health Networking, Applications and Services, 2011, pp. 70-73.

[71] K. Pung, et al., Context-aware middleware for pervasive elderly homecare, IEEE Journal on Selected Areas in Communications 27 (4) (2009) 510-524.

[72] M. Molla, S. Ahamed, A survey of middleware for sensor network and challenges, in: International Conference on Parallel
Processing Workshops, 2006, p. 223-228.

[73] M. Rodriguez, J. Favela, Assessing the salsa architecture for developing agent-based ambient computing applications, Science of Computer Programming 77 (1) (2012) 46-65.

[74] C. Perera, A. Zaslavsky, P. Christen, D. Georgakopoulos, Ca4iot: Context awareness for internet of things, in: International Conference on Green Computing and Communications, 2012, pp. 775-782.

[75] S. Spinsante, E. Gambi, Remote health monitoring by osgi technology and digital tv integration, IEEE Transactions on Consumer Electronics 58 (4) (2012) 1434-1441.

[76] T. Perumal, A. Ramli, C. Leong, S. Mansor, K. Samsudi, Interoperability among heterogeneous systems in smart home environment, in: IEEE International Conference on Signal Image Technology and Internet Based Systems, 2008, pp. 177-186.

[77] H. Martin, A. Bernardos, L. Bergesio, P. Tarrio, Analysis of key aspects to manage wireless sensor networks in ambient assisted living environments, in: nternational Symposium on Applied Sciences in Biomedical and Communication Technologies, 2009, pp. 1-8.

[78] W. Lin, Y. Sheng, Using osgi upnp and zigbee to provide a wireless ubiquitous home healthcare environment, in: International Conference on Mobile Ubiquitous Computing, Systems, Services and Technologies, 2008, pp. 268-273.

[79] Q. Lin, H. Ni, X. Zhou, An osgi-based heath service platform for elderly people, in: International Conference on e-Health Networking, Applications and Services, 2012, pp. 317-320.

[80] S. Nourizadeh, C. Deroussent, Y. Song, J. Thomesse, A distributed elderly healthcare system, in: Mobilizing Health Information to Support Healthcare-related Knowledge Work, 2009, pp. 59-68.

[81] I. Chen, C. Tsai, Pervasive digital monitoring and transmission of pre-care patient biostatics with an osgi, mom and soa based remote health care system, in: IEEE International Conference on Pervasive Computing and Communications, 2008, pp. 704709.

[82] T. Lemlouma, S. Laborie, P. Roose, Toward a context-aware and automatic evaluation of elderly dependency in smart homes and cities, in: IEEE International Symposium on a World of Wireless Mobile and Multimedia Networks, 2013, pp. $1-6$.

[83] L. Atallah, G. Yang, The use of pervasive sensing for behaviour profiling - a survey, Pervasive and Mobile Computing 5 (5) (2009) 447-464.

[84] H. Mshali, T. Lemlouma, D. Magoni, Analysis of dependency evaluation models for ehealth services, in: IEEE Global Communications Conference, 2014, pp. 2429-2435.

[85] S. Katz, A. Ford, R. Moskowitz, B. Jackson, M. Jaffe, Studies of illness in the aged, the index of adl: a standardized measure of biological and psychosocial function, Journal of the American Medical Association 185 (12) (1963) 914-919.

[86] M. Lawton, E. Brody, Assessment of older people: Self maintaining and instrumental activities of daily living, Nursing Research 19 (3) (1970) 179-186.

[87] D. Trabelsi, S. Mohammed, F. Chamroukhi, L. Oukhellou, Y. Amirat, An unsupervised approach for automatic activity recognition based on hidden markov model regression, IEEE Transactions on Automation Science and Engineering 10 (3) (2013) 829-835.

[88] S. Chernbumroong, S. Cang, H. Yu, A practical multi-sensor activity recognition system for home-based care, Decision Support Systems 66 (2014) 61-70.

[89] S. Chernbumroong, S. Cang, A. Atkins, H. Yu, Elderly activities recognition and classification for applications in assisted living, Expert Systems with Applications 40 (5) (2013) 16621674

[90] H. Hsu, C. Chen, Rfid-based human behavior modeling and anomaly detection for elderly care, Mobile Information Systems 6 (4) (2010) 341-354.

[91] O. Brdiczka, J. Crowley, P. Reignier, Learning situation models in a smart home, IEEE Transactions on Systems, Man, and 
Cybernetics, Part B: Cybernetics 39 (1) (2009) 56-63.

[92] Q. X. S. Chatterjee, K. Dutta, A predictive modeling engine using neural networks: Diabetes management from sensor and activity data, in: International Conference on e-Health Networking, Applications and Services, 2012, pp. 230-237.

[93] A. Forkan, I. Khalil, Z. Tari, S. Foufou, A. Bouras, A contextaware approach for long-term behavioural change detection and abnormality prediction in ambient assisted living, Pattern Recognition 48 (3) (2015) 628-641.

[94] E. Kim, S. Helal, D. Cook, Human activity recognition and pattern discovery, IEEE Pervasive Computing 9 (1) (2010) 4853.

[95] L. Chen, I. Khalil, Activity recognition: Approaches, practices and trends, in: Activity Recognition in Pervasive Intelligent Environments, Springer, 2011, pp. 1-31.

[96] L. Ye, Z. Qin, J. Wang, J. Jin, Anomaly event detection in temporal sensor network data of intelligent environments, in: International Conference on Computer Engineering and Technology, Vol. 7, 2010, pp. 414-420.

[97] V. Chandola, A. Banerjee, V. Kumar, Anomaly detection: A survey, ACM Comput. Surv. 41 (3) (2009) 15:1-15:58.

[98] S. Zhang, S. McClean, B. Scotney, X. Hong, C. Nugent, M. Mulvenna, Decision support for alzheimer's patients in smart homes, in: IEEE International Symposium on Computer-Based Medical Systems, 2008, pp. 236-241.

[99] V. Rialle, J. Lamy, N. Noury, L. Bajolle, Telemonitoring of patients at home: a software agent approach, Computer Methods and Programs in Biomedicine 72 (3) (2003) 257-268.

[100] K. Henricksen, J. Indulska, A software engineering framework for context-aware pervasive computing, in: IEEE Annual Conference on Pervasive Computing and Communications, 2004, pp. $77-86$.

[101] X. W. D. Zhang, T. Gu, Enabling context-aware smart home with semantic web technologies, International Journal of Human-friendly Welfare Robotic Systems 6 (4) (2005) 1220.

[102] B. Bouchard, S. Giroux, A. Bouzouane, A smart home agent for plan recognition of cognitively-impaired patients., Journal of Computers 1 (5) (2006) 53-62.

[103] D. Zhang, H. Huang, C. Lai, X. Liang, Q. Zou, M. Guo, Survey on context-awareness in ubiquitous media, Multimedia tools and applications 67 (1) (2013) 179-211.

[104] X. Wang, D. Zhang, T. Gu, H. Pung, Ontology based context modeling and reasoning using owl, in: IEEE Annual Conference on Pervasive Computing and Communications Workshops, 2004, pp. 18-22.

[105] L. Chen, C. Nugent, H. Wang, A knowledge-driven approach to activity recognition in smart homes, IEEE Transactions on Knowledge and Data Engineering 24 (6) (2012) 961-974.

[106] A. Bikakis, T. Patkos, G. Antoniou, D. Plexousakis, A survey of semantics-based approaches for context reasoning in ambient intelligence, in: Constructing ambient intelligence, Springer, 2008, pp. 14-23.

[107] L. Wang, T. Gu, X. Tao, H. Chen, J. Lu, Recognizing multiuser activities using wearable sensors in a smart home, Pervasive and Mobile Computing 7 (3) (2011) 287-298.

[108] P. Chung, C. Liu, A daily behavior enabled hidden markov model for human behavior understanding, Pattern Recognition 41 (5) (2008) 1572-1580.

[109] T. Duong, H. Bui, D. Phung, S. Venkatesh, Activity recognition and abnormality detection with the switching hidden semi-markov model, in: IEEE Computer Society Conference on Computer Vision and Pattern Recognition, Vol. 1, IEEE, 2005, pp. 838-845.

[110] Z. Liouane, T. Lemlouma, P. Roose, F. Weis, M. Hassani, A Markovian-based Approach for Daily Living Activities Recognition, in: The International Conference on Sensor Networks (SENSORNETS'16), 2016, pp. 214-219.

[111] W. Kang, D. Shin, D. Shin, Detecting and predicting of abnormal behavior using hierarchical markov model in smart home network, in: International Conference on Industrial Engineer- ing and Engineering Management, 2010, pp. 410-414.

[112] G. Fenza, D. Furno, V. Loia, Enhanced healthcare environment by means of proactive context aware service discovery, in: IEEE International Conference on Advanced Information Networking and Applications, 2011, pp. 625-632.

[113] G. Singla, D. Cook, S. Maureen, Recognizing independent and joint activities among multiple residents in smart environments, Journal of ambient intelligence and humanized computing 1 (1) (2010) 57-63.

[114] D. Vail, M. Veloso, J. Lafferty, Conditional random fields for activity recognition, in: Proceedings of international joint conference on Autonomous agents and multiagent systems, ACM, 2007.

[115] H.-S. Park, K. Oh, S.-B. Cho, Bayesian network-based highlevel context recognition for mobile context sharing in cyberphysical system, International Journal of Distributed Sensor Networks 7 (1) (2011) 1-10.

[116] J. Hong, S. Yang, S. Cho, Conamsn: A context-aware messenger using dynamic bayesian networks with wearable sensors, Expert Systems with Applications 37 (6) (2010) 4680-4686.

[117] D. Cook, M. Schmitter-Edgecombe, Assessing the quality of activities in a smart environment, Methods of Information in Medicine 48 (5) (2009) 480-485.

[118] D. Cook, Learning setting-generalized activity models for smart spaces, IEEE Intelligent Systems 27 (1) (2012) 32-38.

119] F. Cardinaux, S. Brownsell, M. Hawley, D. Bradley, Modelling of behavioural patterns for abnormality detection in the context of lifestyle reassurance, in: Progress in Pattern Recognition, Image Analysis and Applications, Springer, 2008, pp. 243-251.

[120] X. Xie, J. Guo, H. Zhang, T. Jiang, R. Bie, Y. Sun, Neuralnetwork based structural health monitoring with wireless sensor networks, in: International Conference on Natural Computation, 2013, pp. 163-167.

121] Z. Jin, J. Oresko, S. Huang, A. Cheng, Hearttogo: A personalized medicine technology for cardiovascular disease prevention and detection, in: IEEE Life Science Systems and Applications Workshop, 2009, pp. 80-83.

[122] A. Pantelopoulos, N. Bourbakis, A health prognosis wearable system with learning capabilities using nns, in: International Conference on Tools with Artificial Intelligence, 2009, pp. 243247.

[123] A. Khan, Y. Lee, S. Lee, T. Kim, A triaxial accelerometerbased physical-activity recognition via augmented-signal features and a hierarchical recognizer, IEEE Transactions on Information Technology in Biomedicine 14 (5) (2010) 1166-1172.

[124] F. Rivera-Illingworth, V. Callaghan, H. Hagras, Towards the detection of temporal behavioural patterns in intelligent environments, in: International Conference on Intelligent Environments, Vol. 1, IET, 2006, pp. 119-125.

$125]$ C. Lee, K. Lim, W. Woon, Statistical and entropy based multi purpose human motion analysis, in: International Conference on Signal Processing Systems, Vol. 1, IEEE, 2010, pp. 734-738.

[126] L. Vintan, A. Gellert, J. Petzold, T. Ungerer, Person movement prediction using neural networks, in: Workshop on Modeling and Retrieval of Context, 2004, pp. 1-12.

[127] C. Tseng, D. Cook, Mining from time series human movement data, in: IEEE International Conference on Systems, Man and Cybernetics, Vol. 4, IEEE, 2006, pp. 3241-3243.

[128] H. Li, Q. Zhang, P. Duan, A novel one-pass neural network approach for activities recognition in intelligent environments, in: World Congress on Intelligent Control and Automation, IEEE, 2008, pp. 50-54.

[129] L. Kau, C. Chen, A smart phone-based pocket fall accident detection, positioning, and rescue system, IEEE Journal of Biomedical and Health Informatics 19 (1) (2015) 44-56.

[130] A. Fleury, M. Vacher, N. Noury, Svm-based multimodal classification of activities of daily living in health smart homes: sensors, algorithms, and first experimental results, IEEE Transactions on Information Technology in Biomedicine 14 (2) (2010) $274-283$. 
[131] Y. Hung, C. Chiang, S. Hsu, C. Chan, Abnormality detection for improving elder's daily life independent, in: Aging Friendly Technology for Health and Independence, Springer, 2010, pp. 186-194.

[132] R. Kadouche, H. Pigot, B. Abdulrazak, S. Giroux, User's behavior classification model for smart houses occupant prediction, in: Activity Recognition in Pervasive Intelligent Environments, Springer, 2011, pp. 149-164.

[133] K. Sha, G. Zhan, W. Shi, M. Lumley, C. Wiholm, B. Arnetz, Spa: A smart phone assisted chronic illness self-management system with participatory sensing, in: International Workshop on Systems and Networking Support for Health Care and Assisted Living Environments, 2008, pp. 1-3.

[134] L. Bao, S. Intille, Activity recognition from user-annotated acceleration data, in: Pervasive computing, Springer, 2004, pp. $1-17$.

[135] K. Htike, O. Khalifa, H. Ramli, M. Abushariah, Human activity recognition for video surveillance using sequences of postures, in: International Conference on e-Technologies and Networks for Development, IEEE, 2014, pp. 79-82.

[136] V. Jakkula, Predictive data mining to learn health vitals of a resident in a smart home, in: IEEE International Conference on Data Mining Workshops, 2007, pp. 163-168.

[137] H.-H. Hsu, K.-C. Lu, X. Takizawa, Abnormal behavior detection with fuzzy clustering for elderly care, in: Computer Symposium (ICS), 2010 International, IEEE, 2010, pp. 6-11.

[138] M. Pourhomayoun, Multiple model analytics for adverse event prediction in remote health monitoring systems, in: IEEE Healthcare Innovation Conference, IEEE, 2014, pp. 106-110.

[139] T. Lin, P. Lin, Performance comparison of indoor positioning techniques based on location fingerprinting in wireless networks, in: International Conference on Wireless Networks, Communications and Mobile Computing, Vol. 2, IEEE, 2005, pp. 1569-1574.

[140] H. Mshali, T. Lemlouma, D. Magoni, A predictive approach for efficient e-health monitoring, in: IEEE International Conference on e-Health Networking, Applications and Services, 2015, pp. $268-273$

[141] V. Osmani, S. Balasubramaniam, D. Botvich, A bayesian network and rule-base approach towards activity inference, in: IEEE Vehicular Technology Conference, 2007, pp. 254-258.

[142] S. Satterfield, T. Reichherzer, J. Coffey, E. El-Sheikh, Application of structural case-based reasoning to activity recognition in smart home environments, in: IEEE International Conference on Machine Learning and Applications, Vol. 1, 2012, pp. $1-6$.

[143] F. Doctor, H. Hagras, V. Callaghan, A. Lopez, An adaptive fuzzy learning mechanism for intelligent agents in ubiquitous computing environments, in: International Conference on $\mathrm{Au}-$ tomation, 2004, pp. 101-106.

[144] H. Medjahed, D. Istrate, J. Boudy, B. Dorizzi, Human activities of daily living recognition using fuzzy logic for elderly home monitoring, in: IEEE International Conference on Fuzzy Systems, IEEE, 2009, pp. 2001-2006.

[145] E. Shimokawara, T. Kaneko, T. Yamaguchi, M. Mizukawa, N. Matsuhira, Estimation of basic activities of daily living using zigbee $3 \mathrm{~d}$ accelerometer sensor network, in: International Conference on Biometrics and Kansei Engineering, 2013, pp. 251-256.

[146] S. Das, P. Ghosh, S. Kar, Hypertension diagnosis: a comparative study using fuzzy expert system and neuro fuzzy system, in: IEEE International Conference on Fuzzy Systems, 2013, pp. 1-7.

[147] B. Yuan, J. Herbert, Context-aware hybrid reasoning framework for pervasive healthcare, Personal and ubiquitous computing 18 (4) (2014) 865-881.

[148] H. Chen, F. Perich, T. Finin, A. Joshi, Soupa: Standard ontology for ubiquitous and pervasive applications, in: International Conference on Mobile and Ubiquitous Systems: Networking and Services, 2004, pp. 258-267.

[149] H. Guermah, T. Fissaa, H. Hafiddi, M. Nassar, A. Kriouile,
Context modeling and reasoning for building context aware services, in: IEEE International Conference on Computer Systems and Applications, 2013, pp. 1-7.

[150] A. Hristoskova, V. Sakkalis, G. Zacharioudakis, M. Tsiknakis, F. Turck, Ontology-driven monitoring of patient's vital signs enabling personalized medical detection and alert, Journal of Sensors 14 (1) (2014) 1598-1628.

[151] D. Riboni, C. Bettini, Cosar: hybrid reasoning for contextaware activity recognition, Personal and Ubiquitous Computing 15 (3) (2011) 271-289.

[152] K. Wongpatikaseree, M. Ikeda, M. Buranarach, Activity recognition using context-aware infrastructure ontology in smart home domain, in: International Conference on Knowledge, Information and Creativity Support Systems, 2012, pp. 50-57.

[153] I. Bae, An ontology-based approach to adl recognition in smart homes, Future Generation Computer Systems 33 (2014) 32-41.

154] W. Dargie, The role of probabilistic schemes in multisensor context-awareness, in: IEEE International Conference on Pervasive Computing and Communications Workshops, IEEE, 2007, pp. 27-32.

[155] H. Zhang, J. Liu, Fault diagnosing ecg in body sensor networks based on hidden markov model, in: International Conference on Mobile Ad-hoc and Sensor Networks, 2014, pp. 123-129.

[156] N. Nguyen, D. Phung, S. Venkatesh, H. Bui, Learning and detecting activities from movement trajectories using the hierarchical hidden markov model, in: IEEE Computer Society Conference on Computer Vision and Pattern Recognition, Vol. 2, 2005, pp. 955-960.

[157] D. Ge, W. Li, L. Wei, An intelligent reasoning machine for the home-based care, in: IEEE International Conference on Electronics, Computers and Artificial Intelligence, 2015, pp. 9-14.

[158] Y. Du, F. Chen, W. Xu, Y. Li, Recognizing interaction activities using dynamic bayesian network, in: 18th International Conference on Pattern Recognition, 2006, pp. 618-621.

[159] E. Tapia, S. Intille, K. Larson, Activity recognition in the home using simple and ubiquitous sensors, Pervasive Computing, Lecture Notes in Computer Science 3001 (2004) 158-175.

[160] J. Parkka, L. Cluitmans, M. Ermes, Personalization algorithm for real-time activity recognition using pda, wireless motion bands, and binary decision tree, IEEE Transactions on Information Technology in Biomedicine 14 (5) (2010) 1211-1215.

[161] M. Alhamid, J. Saboune, A. Alamri, A. E. Saddik, Hamon: An activity recognition framework for health monitoring support at home, in: IEEE Instrumentation and Measurement Technology Conference, 2011, pp. 1-5.

[162] S. Tao, M. Kudo, H. Nonaka, Privacy-preserved behavior analysis and fall detection by an infrared ceiling sensor network, Sensors 12 (12) (2012) 16920-16936.

[163] B. Abdulrazak, R. Yared, Prevent cooking risks in kitchen of elderly people: Adaptable reasoning engine based on fuzzy logic for smart oven, in: IEEE International Conference on Computer and Information Technology; Ubiquitous Computing and Communications; Dependable, Autonomic and Secure Computing; Pervasive Intelligence and Computing, 2015, pp. $2165-2172$.

[164] A. Gellert, L. Vintan, Person movement prediction using hidden markov models, Studies in Informatics and Control 15 (1) (2006) 17-30.

165] S. Rao, D. Cook, Predicting inhabitant action using action and task models with application to smart homes, International Journal on Artificial Intelligence Tools 13 (01) (2004) 81-99.

[166] I. Morsi, Y. A. El Gawad, Zakria, Fuzzy logic in heart rate and blood pressure measuring system, in: IEEE Sensors Applications Symposium (SAS), 2013, 2013, pp. 113-117.

[167] M. Vlaminck, L. Jovanov, P. V. Hese, B. Goossens, W. Philips, A. Pizurica, Obstacle detection for pedestrians with a visual impairment based on 3d imaging, in: International Conference on 3D Imaging, 2013, pp. 1-7.

[168] D. Freitas, T. Marcondes, L. Nakamura, J. Ueyama, P. Gomes, R. Meneguette, Combining cell phones and wsns for prevent- 
ing accidents in smart-homes with disabled people, in: International Conference on New Technologies, Mobility and Security, 2015, pp. 1-5.

[169] L. Yang, Y. Ge, W. Li, W. Rao, W. Shen, A home mobile healthcare system for wheelchair users, in: International Conference on Computer Supported Cooperative Work in Design, IEEE, 2014, pp. 609-614

[170] M. S. Hawley, et al., A voice-input voice-output communication aid for people with severe speech impairment, IEEE Transactions on Neural Systems and Rehabilitation Engineering 21 (1) (2013) 23-31.

[171] C. Lu, L. Fu, Robust location-aware activity recognition using wireless sensor network in an attentive home, IEEE journal on Automation Science and Engineering 6 (4) (2009) 598-609.

[172] N. Suryadevara, S. Mukhopadhyay, Wireless sensor network based home monitoring system for wellness determination of elderly, IEEE Sensors Journal 12 (6) (2012) 1965-1972.

[173] J. Eklund, T. Hansen, J. Sprinkle, S. Sastry, Information technology for assisted living at home: building a wireless infrastructure for assisted living, in: IEEE International Conference on Engineering in Medicine and Biology Society, 2005, pp. 3931-3934.

[174] A. Tabar, A. Keshavarz, H. Aghajan, Smart home care network using sensor fusion and distributed vision-based reasoning, in: International workshop on Video Surveillance and Sensor Networks, 2006, pp. 145-154.

[175] H. Medjahed, D. Istrate, J. Boudy, J. Baldinger, B. Dorizzi, A pervasive multi-sensor data fusion for smart home healthcare monitoring, in: IEEE International Conference on Fuzzy Systems, IEEE, 2011, pp. 1466-1473.

[176] W. Chung, C. Yau, K. Shin, k. Myllyla, A cell phone based health monitoring system with self analysis processor using wireless sensor network technology, in: IEEE International Conference on Engineering in Medicine and Biology Society, 2007, pp. 3705-3708.

[177] M. Raad, L. Yang, A ubiquitous smart home for elderly, journal of Information Systems Frontiers 11 (5) (2009) 529-536.

[178] A. Mateska, M. Pavloski, L. Gavrilovska, Rfid and sensors enabled in-home elderly care, in: IEEE International Convention on Information and Communication Technology, Electronics and Microelectronics, 2011, pp. 285-290.

[179] Q. Zhang, M. Karunanithi, R. Rana, J. Liu, Determination of activities of daily living of independent living older people using environmentally placed sensors, in: IEEE International Conference on Engineering in Medicine and Biology Society, 2013, pp. 7044-7047.

[180] M. Mubashir, L. Shao, L. Seed, A survey on fall detection: Principles and approaches, Neurocomputing 100 (2013) 144152

[181] A. Rakhman, L. Nugroho, Widyawan, Kurnianingsih, Fall detection system using accelerometer and gyroscope based on smartphone, in: International Conference on Information Technology, Computer and Electrical Engineering, 2014, pp. 99-104.

[182] A. Ariani, et al., Design of an unobtrusive system for fall detection in multiple occupancy residences, in: 35th Annual International Conference of the IEEE Engineering in Medicine and Biology Society (EMBC), 2013, pp. 4690-4693.

[183] M. Alwan, et al., A smart and passive floor-vibration based fall detector for elderly, in: IEEE Information and Communication Technologies, Vol. 1, 2006, pp. 1003-1007.

[184] B. T. Nukala, N. Shibuya, A. I. Rodriguez, J. Tsay, T. Q. Nguyen, S. Zupancic, D. Y. C. Lie, A real-time robust fall detection system using a wireless gait analysis sensor and an artificial neural network, in: 2014 IEEE Healthcare Innovation Conference, 2014, pp. 219-222.

[185] S. Kim, Y. Jeong, S. Zark, Rfid-based indoor location tracking to ensure the safety of the elderly in smart home environments, Personal and ubiquitous computing 17 (8) (2013) 1699-1707.

[186] J. Dai, X. Bai, Z. Yang, Z. Shen, D. Xuan, Perfalld: A pervasive fall detection system using mobile phones, in: IEEE
International Conference on Pervasive Computing and Communications Workshops, 2010, pp. 292-297.

187] X. Le, M. Mascolo, A. Gouin, N. Noury, Health smart home for elders a tool for automatic recognition of activities of daily living, in: IEEE International Conference on Engineering in Medicine and Biology Society, 2008, pp. 3316-3319.

[188] J. Wyffels, J. Brabanter, P. Crombez, P. Verhoeve, B. Nauwelaers, L. Strycker, Distributed, signal strength-based indoor localization algorithm for use in healthcare environments, IEEE Journal of Biomedical and Health Informatics 18 (6) (2014) 1887-1893.

189] J. Shin, G. Chung, K. Kim, J. Kim, B. Hwang, K. Park, Ubiquitous house and unconstrained monitoring devices for home healthcare system, in: International Special Topic Conference on Information Technology Applications in Biomedicine, 2007, pp. 201-204.

[190] J. Kim, H. Choi, H. Wang, N. Agoulmine, M. Deen, J. Hong, Postech's u-health smart home for elderly monitoring and support, in: IEEE International Symposium on a World of Wireless Mobile and Multimedia Networks, 2010, pp. 1-6.

191] V. Shnayder, B. Chen, Z. Lorincz, T. Fulford-Jones, M. Welsh, Sensor networks for medical care, in: Conference on Embedded Networked Sensor Systems, Vol. 5, 2005, pp. 314-314.

[192] P. Leijdekkers, V. Gay, E. Lawrence, Smart homecare system for health tele-monitoring, in: International Conference on the Digital Society, 2007, pp. 3-3.

[193] S. Macis, et al., Home telemonitoring of vital signs through a tv-based application for elderly patients, in: IEEE International Symposium on Medical Measurements and Applications, 2015, pp. 169-174.

[194] R. Robles, T. Kim, Review: context aware tools for smart home development, International Journal of Smart Home 4 (1) (2010) 1-12.

[195] H. Mshali, T. Lemlouma, D. Magoni, Context-aware adaptive framework for e-health monitoring, in: IEEE International Conference on Green Computing and Communications (IEEE GreenCom 2015 merged with the IEEE DSDIS2015), 2015, pp. 276-283.

[196] IEA, International Ergonomics Association, What is ergonomics?, Http://www.iea.cc/ [Accessed: June 2016] (2015).

[197] S. Hignett, P. Carayon, P. Buckle, K. Catchpole, State of science: human factors and ergonomics in healthcare, Ergonomics 56 (10) (2013) 1491-1503.

198] M. A. Hossain, Perspectives of human factors in designing elderly monitoring system, Computers in Human Behavior 33 (2014) 63-68.

[199] R. J. Holden, et al., Seips 2.0: a human factors framework for studying and improving the work of healthcare professionals and patients, Ergonomics 56 (11) (2013) 1669-1686.

[200] A. P. Gurses, A. A. Ozok, P. Pronovost, Time to accelerate integration of human factors and ergonomics in patient safety, BMJ Quality \& Safety 21 (4) (2012) 347-351.

[201] A. Ahlan, B. Ahmad, User acceptance of health information technology (hit) in developing countries: A conceptual model, Procedia Technology 16 (2014) 1287-1296.

[202] R. Alnanih, O. Ormandjieva, T. Radhakrishnan, A new methodology (con-info) for context-based development of a mobile user interface in healthcare applications, in: Pervasive Health, Springer, 2014, pp. 317-342.

203] H. Cruz-Sanchez, L. Havet, M. Chehaider, Y. Song, Mpigate: A solution to use heterogeneous networks for assisted living applications, in: International Conference on Ubiquitous Intelligence Computing and International Conference on Autonomic Trusted Computing, 2012, pp. 104-111.

[204] J. Corchado, J. Bajo, D. Tapia, A. Abraham, Using heterogeneous wireless sensor networks in a telemonitoring system for healthcare, IEEE Transactions on Information Technology in Biomedicine 14 (2) (2010) 234-240.

205] Y. E. R. Julio, Design ubiquitous architecture for telemedicine based on mhealth arduino $4 \mathrm{~g}$ lte, in: 18th IEEE International Conference on e-Health Networking, Applications and Services 
(Healthcom), 2016, pp. 1-6.

[206] E. Sarra, H. Moungla, S. Benayoune, A. Mehaoua, Coexistence improvement of wearable body area network (wban) in medical environment, in: IEEE International Conference on Communications, 2014, pp. 5694-5699.

[207] Y. Kim, S. Lee, S. Lee, Coexistence of zigbee-based wban and wifi for health telemonitoring systems, IEEE Journal of Biomedical and Health Informatics 20 (1) (2016) 222-230.

[208] Y. Mao, Z. Zhao, X. Jia, Understanding the indoor interference between ieee 802.15 .4 and ieee $802.11 \mathrm{~b} / \mathrm{g}$ via measurements, in: International Conference on Wireless Communications and Signal Processing, 2011, pp. 1-5.

[209] F. Martelli, R. Verdone, Coexistence issues for wireless body area networks at $2.45 \mathrm{ghz}$, in: European Wireless Conference, 2012, pp. 1-6.

[210] J. Misic, V. Misic, Bridge performance in a multitier wireless network for healthcare monitoring, IEEE Journal on Wireless Communications 17 (1) (2010) 90-95.

[211] S. Silva, S. Soares, T. Fernandes, A. Valente, A. Moreira, Coexistence and interference tests on a bluetooth low energy frontend, in: IEEE Science and Information Conference, 2014, pp. $1014-1018$

[212] A. King, J. Brown, U. Roedig, Dcca: Differentiating clear channel assessment for improved 802.11/802.15. 4 coexistence, in: IEEE International Conference on Wireless and Mobile Computing, Networking and Communications, 2014, pp. 4550 .

[213] N. Torabi, V. Leung, Cross-layer design for prompt and reliable transmissions over body area networks, IEEE Journal of Biomedical and Health Informatics 18 (4) (2014) 1303-1316.

[214] Z. Zou, Y. Bao, F. Deng, H. Li, An approach of reliable data transmission with random redundancy for wireless sensors in structural health monitoring, IEEE Sensors Journal 15 (2) (2015) 809-818.

[215] U. Varshney, Improving wireless health monitoring using incentive-based router cooperation, IEEE Journal of Computer 41 (5) (2008) 56-62.

[216] S. Chen, T. Kao, C. Chan, C. Huang, C. Chiang, C. Lai, T. Tung, P. Wang, A reliable transmission protocol for zigbeebased wireless patient monitoring, IEEE Transactions on Information Technology in Biomedicine 16 (1) (2012) 6-16.

[217] U. Varshney, A framework for supporting emergency messages in wireless patient monitoring, Decision Support Systems 45 (4) (2008) 981-996.

[218] P. Kumar, H. Lee, Security issues in healthcare applications using wireless medical sensor networks: A survey, Journal of Sensors 12 (1) (2011) 55-91.

[219] F. Kargl, E. Lawrence, M. Fischer, Y. Lim, Security, privacy and legal issues in pervasive ehealth monitoring systems, in: International Conference on Mobile Business, 2008, pp. 296304.

[220] K. Zhang, K. Yang, X. Liang, Z. Su, X. Shen, H. H. Luo, Security and privacy for mobile healthcare networks: from a quality of protection perspective, IEEE Wireless Communications 22 (4) (2015) 104-112.

[221] M. Meingast, T. Roosta, S. Sastry, Security and privacy issues with health care information technology, in: IEEE International Conference on Engineering in Medicine and Biology Society, 2006, pp. 5453-5458.

[222] O. Omeni, O. Eljamaly, A. Burdett, Energy efficient medium access protocol for wireless medical body area sensor networks, in: International Summer School and Symposium on Medical Devices and Biosensors, 2007, pp. 29-32.

[223] H. Strey, P. Richman, R. Rozensky, S. Smith, L. Endee, Bluetooth low energy technologies for applications in health care: proximity and physiological signals monitors, in: International Conference and Expo on Emerging Technologies for a Smarter World, 2013, pp. 1-4. 